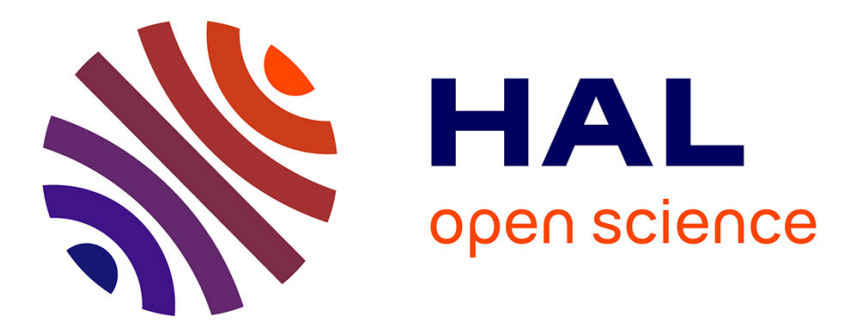

\title{
On the use of a high-order discontinuous Galerkin method for DNS and LES of wall-bounded turbulence
} Marta de La Llave Plata, Vincent Couaillier, Marie-Claire Le Pape

\section{To cite this version:}

Marta de La Llave Plata, Vincent Couaillier, Marie-Claire Le Pape. On the use of a high-order discontinuous Galerkin method for DNS and LES of wall-bounded turbulence. 2016. hal-01402105

\section{HAL Id: hal-01402105 \\ https://hal.science/hal-01402105}

Preprint submitted on 29 Nov 2016

HAL is a multi-disciplinary open access archive for the deposit and dissemination of scientific research documents, whether they are published or not. The documents may come from teaching and research institutions in France or abroad, or from public or private research centers.
L'archive ouverte pluridisciplinaire HAL, est destinée au dépôt et à la diffusion de documents scientifiques de niveau recherche, publiés ou non, émanant des établissements d'enseignement et de recherche français ou étrangers, des laboratoires publics ou privés. 


\title{
On the use of a high-order discontinuous Galerkin method for DNS and LES of wall-bounded turbulence
}

\author{
Marta de la Llave Plata ${ }^{\mathrm{a}, *}$, Vincent Couaillier ${ }^{\mathrm{a}}$, Marie-Claire le Pape ${ }^{\mathrm{a}}$ \\ ${ }^{a}$ ONERA - The French Aerospace Lab, FR-92322 Châtillon, France
}

\begin{abstract}
We assess the performance of a high-order discontinuous Galerkin (DG) approach to simulate wall-bounded turbulent flows for a range of Reynolds numbers. First, a plane channel flow configuration with turbulent heat transfer at $R e_{\tau}=640$ is considered. Second, a detached flow configuration is investigated. This configuration represents the flow over periodically arranged hills. Four bulk Reynolds numbers $R e_{b}$ are considered: 2800, 10595, 19000 , and 37000 . For $R e_{b}=2800$ direct numerical simulation (DNS) is used. Large-eddy simulations (LES) based on the WALE subgrid-scale model are carried out for the higher $R e_{b}$. The simulation results are compared to reference data from CFD and experiment. $h p$-convergence analyses are performed which demonstrate the superior performance of increasing the polynomial order as compared to refining the mesh. It appears from this work that the use of a subgrid modelling approach together with local hp-adaptation could greatly improve the accuracy of the solution without penalising the computational cost of the simulation.
\end{abstract}

Keywords: Discontinuous Galerkin method, large-eddy simulation, direct numerical simulation, wall-bounded turbulence

\section{Introduction}

The development of mathematical and numerical tools for the solution of the Navier-Stokes (N-S) equations in the turbulent flow regime is a topic of acknowledged practical importance to scientists and engineers. The pioneering work of Orszag and Patterson in 1972 [1], proved that it is possible to perform computer simulations of fully developed turbulent flows, called direct numerical simulations (DNS). DNS provides a deterministic time-dependent numerical solution of the fluid flow equations, in which all the scales of motion are accurately resolved. Unfortunately, DNS is computationally very expensive, and is restricted to low-to-moderate Reynolds numbers. This fact has motivated the

\footnotetext{
${ }^{*}$ Corresponding author

Email address: marta.de_la_llave_plata@onera.fr (Marta de la Llave Plata)
} 
scientific community to explore alternative approaches capable of reducing the number of degrees of freedom (DOFs) necessary to resolve the turbulence.

The large-eddy simulation (LES) approach provides a way of reducing the size of the numerical problem at hand by solving explicitly only for the largescale motions while modelling the effect of the small (or subgrid) scales on the resolved field. The separation of scales between large and small can be achieved either by applying a spatial filter to the N-S equations or by means of a projection operation $[2,3]$.

The accurate and efficient simulation of turbulent flows imposes two main requirements on the discretization scheme. First, it must be able to provide very low levels of dissipation and dispersion. The aim is to resolve accurately over long integration periods the finest scales of turbulence that can be represented on the computational grid. Second, it must be highly parallelizable so that we can achieve high parallel performance on many-core architectures.

The high-order discontinuous Galerkin (DG) method $[4,5,6,7]$ meets these two requirements, i.e. high-order accuracy and excellent parallel capabilities [8]. This is the reason why the scientific community is showing a growing interest in this type of approach $[9,10]$.

The DG method is based on the variational projection of the N-S equations onto a hierarchy of polynomial basis functions. They combine some features of finite volume (FV) methods and finite element (FE) methods. It is characterised by being locally conservative and by providing high-order spectral accuracy not only in the interior of the computational domain but also at the physical boundaries, and on irregular meshes. Another attractive feature of the DG method is the possibility to enforce boundary conditions weakly, which has been shown by a number of authors to improve the quality of the solution [11, 12, 13]. Modal DG approximations also provide a natural framework for the implementation of adaption algorithms based on local $p$-refinement, by locally increasing (or decreasing) the polynomial order within the elements. Furthermore, the compacity of the DG scheme greatly simplifies the implementation of h-refinement techniques involving hanging nodes [14].

The overall flexibility provided by the DG approach therefore makes this type of discretization a very appealing tool for the simulation of inhomogeneous high-Reynolds-number flows.

A small number of (well-resolved) DNS computations using DG have been reported in the literature. We can cite the work of Wei and Pollard $[15,16]$ who applied the DG method to study the compressible turbulent channel flow for a range Mach numbers. In $[17,18]$ Chapelier et al. have used the DG method to perform DNS of freely decaying and wall-bounded turbulent flows, highlighting the benefits of the DG approach over traditional numerical schemes in terms of number of DOFs. Diosady et al. [19, 20] have also performed DNS simulations of a number of turbulent flow configurations. In [19], the authors demonstrate the superiority of high-order over low-order methods at equal number of DOFs. In [21], Bassi et al. use an implicit high-order DG method to perform a DNS of the incompressible flow past a sphere at $R e_{D}=1000$.

A greater number of under-resolved DNS, often also called no-model or im- 
plicit LES (ILES), computations can be found in the recent literature (see e.g. $[21,22,23,24,25,26,27,28])$. In the absence of an explicit subgrid-scale model, the philosophy behind ILES is to exploit the dissipation properties of the DG scheme to achieve a stable simulation. In order to reduce the numerical errors due to aliasing when very coarse meshes are used, some authors propose to use additional stabilisation techniques, such as polynomial de-aliasing (over-integration) or polynomial filtering (see e.g. [29, 30, 22]).

As regards to the application of the DG method to LES based on subgridscale modelling we can cite a number of works. Van Der Bos et al. [31] have performed LES of homogeneous isotropic turbulence (HIT) at low Reynolds number using the DG approach. They performed a parametric study in terms of the approximation order, the type of convective flux function and the value of the Smagorinsky constant. They found the best results when using the Smagorinsky model and a high order of accuracy. Marek et al. [32] have performed an LES of an incompressible free round jet at $R e_{D}=20000$ using an eddy-viscosity approach. Abbà et al. [33] have developed an anisotropic dynamic model in the context of a high-order DG method for compressible LES. To this end, the authors exploit the hierarchical nature of the FE basis to define the grid and test filters via projection operators. In [34], Chapelier et al. have proposed a variational multiscale approach based on a modal DG method and applied it to the Taylor-Green vortex configuration at $R e=3000$. Finally, Beck et al. [35] have studied the influence of over-integration on the performance of subgridscale models. The authors demonstrate the benefits of using de-aliasing when a subgrid-scale closure is used at high polynomial degrees.

In this work we will focus on the use of a high-order modal DG approach to perform DNS and LES of two wall-bounded flow configurations for a range of Reynolds numbers. Firstly, under-resolved DNS simulations of a turbulent plane channel flow configuration with heat transfer at $R e_{\tau}=640$ are performed. The adopted strategy consists in modifying the mesh size and the polynomial degree so that the number of DOFs remains constant. The results from the DG simulations are compared against the reference DNS data available in [36].

A detached flow configuration, the periodic flow over a $2 \mathrm{D}$ hill, is also investigated. This configuration represents the flow over periodically arranged hills. This test case is of interest for the evaluation of high-order methods in the context of DNS and LES, due to the periodic boundary conditions and the 2D character of the geometry which significantly reduces the computational cost. A number of DG simulations of this configuration at $R e_{b}=2800$ and 10595 have been reported in the recent literature (see e.g. [19, 26, 33]). Here, we extend the study by also considering the higher Reynolds numbers $R e_{b}=19000$ and 37000 .

Four different bulk Reynolds numbers, $R e_{b}$ based on the bulk velocity at the hill crest $u_{b}$ and the hill height $h$, are therefore considered: 2800, 10595,19000 , and 37000 . The lowest Reynolds number case is computed using DNS and the three other cases are computed using LES based on the WALE subgrid-scale model [37]. An additional no-model LES of the $R e_{b}=10595$ configuration is performed and the results are compared to the corresponding model-based LES. 
The DG solutions are compared to the CFD data provided by Breuer et al. [38] $\left(R e_{b}=2800\right.$ and $\left.R e_{b}=10,595\right)$ using the second-order FV code LESOCC on a curvilinear grid and by Manhart et al. [39] $\left(R e_{b}=37,000\right)$ using the secondorder FV code MGLET on a Cartesian mesh. All LES results are also compared to the experimental data provided by Rapp [40].

This work is aimed at demonstrating the capability of the DG method to produce accurate results for physically complex problems with a reduced number of DOFs. We also highlight the beneficial effect of employing an LES subgrid modelling approach (as opposed to a no-model approach) on coarse meshes when we move towards higher Reynolds numbers.

\section{Governing equations}

The equations governing the evolution of a Newtonian fluid are the N-S equations

$$
\frac{\partial \mathbf{U}}{\partial t}+\nabla \cdot\left(\mathbf{f}_{\mathbf{c}}-\mathbf{f}_{\mathbf{v}}\right)=\mathbf{S}
$$

where, $\mathbf{U}=(\rho, \rho \mathbf{V}, \rho E)^{T}$ is the vector of conservative variables. The vectors $\mathbf{f}_{\mathbf{c}}$ and $\mathbf{f}_{\mathbf{v}}$ are the convective and viscous fluxes, respectively,

$$
\mathbf{f}_{\mathbf{c}}=(\rho \mathbf{V}, \rho \mathbf{V} \otimes \mathbf{V}+p \overline{\bar{I}},(\rho E+p) \mathbf{V})^{T} \quad \mathbf{f}_{\mathbf{v}}=(0, \overline{\bar{\tau}}, \overline{\bar{\tau}} \cdot \mathbf{V}-\mathbf{q})^{T}
$$

and $\mathbf{S}$ is a source term. In (2) $\rho$ represents the density, $E$ the total energy per unit mass, $\mathbf{V}$ is the velocity vector, and $\otimes$ denotes the tensor product. The total energy per unit volume is given by

$$
\rho E=\frac{p}{\gamma-1}+\frac{1}{2} \rho\|\mathbf{V}\|^{2}
$$

where the adiabatic index $\gamma=\frac{c_{p}}{c_{v}}$ is the ratio of specific heat capacities at constant pressure and constant volume conditions, respectively. The static pressure $p$ is related to the static temperature $T$ through the equation of state $p=\rho R T$. Here the gas constant is defined as $R=c_{p}-c_{v}$. The stress tensor $\overline{\bar{\tau}}$ is related to the velocity gradients by the Stokes relations

$$
\overline{\bar{\tau}}=\mu\left[\frac{1}{2}\left(\nabla \mathbf{V}+\nabla \mathbf{V}^{T}\right)-\frac{2}{3} \nabla \cdot \mathbf{V} \overline{\bar{I}}\right]
$$

and the heat-flux vector is given by

$$
\mathbf{q}=-\kappa \nabla T
$$

where $\kappa=\frac{c_{p} \mu}{P r}$ is the thermal conductivity. The Prandtl number $P r$ is assumed to be constant and equal to 0.71 . The Sutherland law provides an expression for the dynamic viscosity $\mu$ as a function of the temperature. 


\section{LES modelling approach}

In this section, the LES approach adopted in this work is briefly outlined. For a detailed description of the compressible LES formalism used in this research the reader is referred to $[41,42,34]$.

In what follows we use Favre (or density-weighted) filtering to define a resolved quantity $\tilde{\phi}$ in terms of the resolved (projected) conservative variables,

$$
\tilde{\phi}=\frac{\overline{\rho \phi}}{\bar{\rho}}
$$

In order to model the effect of the unresolved scales on the resolved field, a subgrid-scale stress term $\tau_{i j}^{s g s}$ is added to the momentum equations. We make use of an eddy-viscosity assumption to express this term as a function of the known resolved field, namely,

$$
\tau_{i j}^{s g s}=\bar{\rho} \nu_{t}\left(2 \tilde{S}_{i j}-\frac{2}{3} \tilde{S}_{k k} \delta_{i j}\right) \quad ; \quad \tilde{S}_{i j}=\frac{1}{2}\left(\frac{\partial \tilde{u}_{i}}{\partial x_{j}}+\frac{\partial \tilde{u}_{j}}{\partial x_{i}}\right)
$$

where $\bar{\rho}$ is the resolved density, $\nu_{t}$ is the eddy viscosity, and $\tilde{S}_{i j}$ denotes the resolved rate-of-strain tensor. In (7), $\tilde{u}_{i}$ denotes the i-th component of the velocity vector $\mathbf{V}$. Based on the same hypothesis considered by Ducros et al. [43] and Lesieur et al. [41] a subgrid heat-flux vector term is also added to the energy equation,

$$
Q_{i}=\bar{\rho} c_{p} \frac{\nu_{t}}{P r_{t}} \frac{\partial \tilde{T}}{\partial x_{i}}
$$

in which $\mathrm{Pr}_{t}$ is the turbulent Prandtl number which is set to 0.6 .

The LES simulations presented in this paper have been performed using the Wall-Adapting Local Eddy-viscosity (WALE) model proposed by Nicoud and Ducros [37]. This model is based on a tensor invariant and is able to represent the proper scaling at the wall, $\nu_{t}=O\left(y^{3}\right)$. In the WALE approach, the eddy viscosity $\nu_{t}$ is expressed as

$$
\nu_{t}=\left(C_{W} \Delta\right)^{2} \frac{\left(\tilde{S}_{i j}^{d} \tilde{S}_{i j}^{d}\right)^{\frac{3}{2}}}{\left(\tilde{S}_{i j} \tilde{S}_{i j}\right)^{\frac{5}{2}}+\left(\tilde{S}_{i j}^{d} \tilde{S}_{i j}^{d}\right)^{\frac{5}{4}}}
$$

where $\tilde{S}_{i j}^{d}$ is defined as

$$
\tilde{S}_{i j}^{d}=\frac{1}{2}\left(\tilde{g}_{i j}^{2}+\tilde{g}_{j i}^{2}\right)-\frac{1}{3} \delta_{i j} \tilde{g}_{k k}^{2}
$$

and it represents the symmetric part of the tensor $\tilde{g}_{i j}^{2}=\tilde{g}_{i k} \tilde{g}_{k j}$, where $\tilde{g}_{i j}=$ $\partial \tilde{u}_{i} / \partial x_{j}$. The filter width $\Delta$ is defined in terms of the DG discretization parameters $h$ and $p$ as $\Delta=h /(p+1)$, and the model constant takes a value of $0.55 \leq C_{W} \leq 0.6$ as recommended in [37]. 


\section{The discontinuous Galerkin method}

The simulation results presented here have been performed using the highorder DG solver Aghora [44, 18] developed at Onera. This solver is designed to solve the full set of compressible N-S equations on unstructured grids.

The DG discretization used in this work is based on a modal approach that relies on the use of a hierarchy of orthogonal polynomial functions as basis for the Galerkin projection. The modal basis used is that proposed in [45] by Bassi et al. The discrete orthogonal polynomial spaces are directly computed in physical space. The methodology consists in defining a starting set of monomial basis functions in each (arbitrarily shaped) element and applying a modified Gram-Schmidt orthogonalization procedure. This ensures the orthogonality of the basis, and thereby the diagonality of the mass matrix. Numerical integration is efficiently performed by means of a Gaussian quadrature. The time integration is performed by using a strong stability preserving third-order Runge-Kutta scheme [46]. The DG method implemented in the Aghora solver is briefly outlined below.

We start by defining a shape-regular partition of the domain $\Omega$, into $N$ nonoverlapping and non-empty cells $\kappa$ of characteristic size $h$.We also define the sets $\mathcal{E}_{i}$ and $\mathcal{E}_{b}$ of interior and boundary faces in $\Omega_{h}$, such that $\mathcal{E}_{h}=\mathcal{E}_{i} \cup \mathcal{E}_{b}$.

Let $\mathcal{V}_{h}^{p}=\left\{\phi \in L^{2}\left(\Omega_{h}\right):\left.\phi\right|_{\kappa} \in \mathcal{P}^{p}(\kappa), \forall \kappa \in \Omega_{h}\right\}$ be the functional space of piecewise polynomials of degree at most $p$, and $\left(\phi_{\kappa}^{1}, \ldots, \phi_{\kappa}^{N_{p}}\right) \in \mathcal{P}^{p}(\kappa)$ a hierarchical and orthonormal modal basis of $\mathcal{V}_{h}^{p}$, of dimension $N_{p}$, confined to $\kappa$. The solution in each element is thus expressed as

$$
\mathbf{u}_{h}(\mathbf{x}, t)=\sum_{l=1}^{N_{p}} \phi_{\kappa}^{l}(\mathbf{x}) \mathbf{U}_{\kappa}^{l}(t), \quad \forall \mathbf{x} \in \kappa, \kappa \in \Omega_{h}, \forall t \geq 0,
$$

in which the polynomial coefficients $\left(\mathbf{U}_{\kappa}^{l}\right)_{1 \leq l \leq N_{p}}$ represent the DOFs of the discrete problem in element $\kappa$. The semi-discrete variational form of system of equations (1) thus reads: find $\mathbf{u}_{h}$ in $\mathcal{V}_{h}^{p}$ such that $\forall \phi_{h} \in \mathcal{V}_{h}^{p}$ we have

$$
\int_{\Omega_{h}} \phi_{h} \partial_{t} \mathbf{u}_{h} d V+\mathcal{L}_{c}\left(\mathbf{u}_{h}, \phi_{h}\right)+\mathcal{L}_{v}\left(\mathbf{u}_{h}, \phi_{h}\right)=\int_{\Omega_{h}} \phi_{h} \mathbf{S}_{h}
$$

In Eqn. (12) $\mathcal{L}_{c}$ and $\mathcal{L}_{v}$ represent the variational projection of the convective and viscous terms, respectively, onto the functional space $\mathcal{V}_{h}^{p}$. Similarly, the right-hand-side of Eqn. (12) is the variational projection of the source term $\mathbf{S}$ onto $\mathcal{V}_{h}^{p}$.

We now introduce the following notation: for a given interface $e$ in $\mathcal{E}_{i}$ we define the average operator $\{u\}=\left(u^{+}+u^{-}\right) / 2$ and the jump operator $\llbracket u \rrbracket=u^{+} \mathbf{n}^{+}-u^{-} \mathbf{n}^{-}$, where $u^{+}$and $u^{-}$are the traces of the variable $u$ at the interface between elements $\kappa^{+}$and $\kappa^{-}$(see Fig. 1). The DG discretization of 


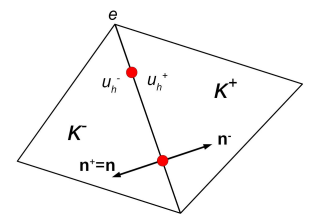

Figure 1: Trace of a variable on a face.

the convective terms then reads

$$
\begin{aligned}
\mathcal{L}_{c}\left(\mathbf{u}_{h}, \phi_{h}\right)= & -\int_{\Omega_{h}} \mathbf{f}_{c}\left(\mathbf{u}_{h}\right) \cdot \nabla_{h} \phi_{h} d V \\
& +\int_{\mathcal{E}_{i}} \llbracket \phi_{h} \rrbracket \mathbf{h}_{c}\left(\mathbf{u}_{h}^{+}, \mathbf{u}_{h}^{-}, \mathbf{n}\right) d S+\int_{\mathcal{E}_{b}} \phi_{h}^{+} \mathbf{f}_{c}\left(\mathbf{u}_{b}\left(\mathbf{u}_{h}^{+}, \mathbf{n}\right)\right) \cdot \mathbf{n} d S
\end{aligned}
$$

The numerical flux $\mathbf{h}_{c}$ is chosen so that it is consistent and compact. For the simulations presented in this paper the local Lax-Friedrichs (LLF) flux has been employed.

$$
\begin{array}{r}
\mathbf{h}_{c}\left(\mathbf{u}_{h}^{+}, \mathbf{u}_{h}^{-}, \mathbf{n}\right)=\left\{\mathbf{f}_{c}\left(\mathbf{u}_{h}\right)\right\}+\alpha \frac{\llbracket \mathbf{u}_{h} \rrbracket}{2} \\
\alpha=\max \left\{\rho_{s}\left(\frac{\partial\left(\mathbf{f}_{c}(\mathbf{u}) \cdot \mathbf{n}\right)}{\partial \mathbf{u}}\right): \mathbf{u}=\mathbf{u}_{h}^{ \pm}\right\}
\end{array}
$$

in which $\frac{\partial}{\partial \mathbf{u}}\left(\mathbf{f}_{c}(\mathbf{u}) \cdot \mathbf{n}\right)$ is the Jacobian matrix of the convective fluxes in the $\mathbf{n}$ direction and $\rho_{s}$ is the spectral radius of the Jacobian matrix. The discretization of the viscous terms is performed using the symmetric interior penalty (SIP) method [47], described in [48],

$$
\begin{aligned}
& \mathcal{L}_{v}\left(\mathbf{u}_{h}, \phi_{h}\right)=\int_{\Omega_{h}} \mathbf{f}_{v}\left(\mathbf{u}_{h}, \nabla_{h} \mathbf{u}_{h}\right) \cdot \nabla_{h} \phi_{h} d V \\
& -\int_{\mathcal{E}_{i}} \llbracket \phi_{h} \rrbracket\left\{\mathbf{f}_{v}\left(\mathbf{u}_{h}, \nabla_{h} \mathbf{u}_{h}\right)\right\} \cdot \mathbf{n} d S-\int_{\mathcal{E}_{b}} \phi_{h}^{+} \mathbf{f}_{v}\left(\mathbf{u}_{b}\left(\mathbf{u}_{h}^{+}, \mathbf{n}\right), \nabla \mathbf{u}_{b}\left(\mathbf{u}_{h}^{+}, \mathbf{n}\right)\right) \cdot \mathbf{n} d S \\
& \left.-\int_{\mathcal{E}_{i}} \llbracket \mathbf{u}_{h} \rrbracket\left\{\mathbf{G}^{T}\left(\mathbf{u}_{h}\right) \nabla_{h} \phi_{h}\right\} \cdot \mathbf{n} d S-\int_{\mathcal{E}_{b}}\left(\mathbf{u}_{h}^{+}-\mathbf{u}_{b}\left(\mathbf{u}_{h}^{+}, \mathbf{n}\right)\right)\left\{\mathbf{G}^{T}\left(\mathbf{u}_{b}\right) \nabla_{h} \phi_{h}^{+}\right)\right\} \cdot \mathbf{n} d S \\
& +\int_{\mathcal{E}_{i}} \eta_{I P} \llbracket \mathbf{u}_{h} \rrbracket \llbracket \phi_{h} \rrbracket d S+\int_{\mathcal{E}_{b}} \eta_{I P}\left(\mathbf{u}_{h}^{+}-\mathbf{u}_{b}\left(\mathbf{u}_{h}^{+}, \mathbf{n}\right)\right) \phi_{h}^{+} d S
\end{aligned}
$$

where $\mathbf{G}=\partial \mathbf{f}_{v} / \partial\left(\nabla_{h} \mathbf{u}_{h}\right)$ is the so-called homogeneity tensor. The penalty parameter $\eta_{I P}$ has to be chosen sufficiently large to ensure the coercivity of the bilinear form and thus the numerical stability of the simulation [47]. 


\section{Turbulent heat transfer in a plane channel at $R e_{\tau}=640$}

Following the work of Abe et al. [49] we consider a fully developed turbulent flow in a plane channel with a passive scalar field representing the temperature in an incompressible flow. The size of the computational domain is $12.8 h \times 2 h \times$ $6.4 h$, where $h$ is half the height of the channel. Periodicity and homogeneity of the flow is assumed in the streamwise and spanwise directions. To study the heat transfer in the channel, the authors consider an additional equation governing the transport of the temperature

$$
\frac{\partial \rho \theta}{\partial t}+\nabla \cdot(\rho \theta \mathbf{u})-\nabla \cdot\left(\frac{\kappa}{c_{p}} \nabla \theta\right)=f_{\theta}
$$

The temperature $\theta$ thus behaves as a passive scalar and $f_{\theta}$ is a source term that ensures a constant heat-flux at the wall $Q_{w}$.

Among the different simulations reported in [49], we focus on the DNS at Reynolds number $R e_{\tau}=640$ and Prandtl number $\operatorname{Pr}=0.71$, performed using an incompressible solver on a grid composed of $1024 \times 256 \times 1024$ nodes.

We also define the bulk Reynolds number based on the bulk velocity $u_{b}$ as

$$
R e_{b}=\frac{u_{b} 2 h}{\nu}=24428 \quad u_{b}=\frac{1}{2 h} \int_{0}^{2 h} u_{1}(y) d y
$$

A forcing term is introduced in the x-momentum equation, $-\left(\nabla_{x} p\right)_{o}$, and in the energy equation, $-u\left(\nabla_{x} p\right)_{o}$. The pressure gradient $\left(\nabla_{x} p\right)_{o}$ is considered spatially constant and ensures a target mass flow rate $\dot{m}_{0}$ corresponding to the specified $R e_{b}$. Thus, the value of the $R e_{\tau}$ is an outcome of the simulation that will allow us to assess the accuracy of the employed numerical approach. Based on the algorithm proposed by Deschamps [50] in the incompressible case, and extended to the compressible case by Lenormand et al. [51, 52] the pressure gradient is computed at each time step as

$$
\left(\nabla_{x} p\right)_{o}^{n+1}=\left(\nabla_{x} p\right)_{o}^{n}+\frac{1}{L_{y} L_{z} \Delta t}\left(\dot{m}_{0}-2 \dot{m}^{n}+\dot{m}^{n-1}\right)
$$

where $\dot{m}^{n}$ is the mass flow rate computed at time step $n$, and $L_{y}$ and $L_{z}$ represent the dimensions of the computational domain in the normal and spanwise directions, respectively.

To maintain consistency with the work presented in [49], Eqn. (18) is solved at each time-step by using the DG approach presented in Sec. 4. By doing so we obtain a scalar field devoid of compressibility effects and therefore a truthful comparison with the DNS results reported in [49] can be made. The source term $f_{\theta}$ is defined in terms of the imposed wall heat-flux ${ }^{1} Q_{w}$ as

$$
f_{\theta}=-\rho u_{1}\left(\nabla_{x} \theta\right)_{o} \quad ; \quad\left(\nabla_{x} \theta\right)_{o}=-\frac{Q_{w}}{c_{p}(\rho u)_{b} h}
$$

\footnotetext{
${ }^{1}$ The heat-flux associated with the passive scalar $Q_{w}$ should not be mistaken for the heatflux q that appears in the compressible energy equation.
} 


\begin{tabular}{cccc} 
Simulation & Order $(p+1)$ & \# Elements & \# DOFs \\
\hline Abe et al. $[49]$ & 4 & $1024 \times 256 \times 1024$ & $268.4 \mathrm{M}$ \\
\hline$D G-p 2$ & 3 & $84 \times 22 \times 84$ & $4.19 \mathrm{M}$ \\
\hline$D G-p 3$ & 4 & $64 \times 16 \times 64$ & $4.19 \mathrm{M}$ \\
\hline$D G-p 4$ & 5 & $52 \times 12 \times 52$ & $4.06 \mathrm{M}$ \\
\hline
\end{tabular}

Table 1: Computational grids.

\begin{tabular}{cccc} 
Simulation & $\Delta t\left(u_{b} / h\right) / 10^{-4}$ & CPU time $/ t c$ & $R e_{\tau}$ \\
\hline$D G-p 2$ & 5.0 & 1.00 & 619 \\
\hline$D G-p 3$ & 8.0 & 1.16 & 676 \\
\hline$D G-p 4$ & 6.7 & 1.36 & 647 \\
\hline
\end{tabular}

Table 2: Computational details for the plane channel flow DG simulations.

\subsection{Computational details}

Under-resolved DNS computations of the configuration described above have been performed using the Aghora DG solver. Three different levels of $h p$ refinement have been considered in this study, for which the number of DOFs is the same and equal to approximately 4.2 million (see Table 1 ). This resolution is four times coarser in each space direction than that used in the reference DNS. To ensure the stability of the simulation at this coarse resolution, overintegration is used as de-aliasing technique by considering an extra-quadrature point in each spatial direction. For a $p t h$-order simulation we thus have $(p+1)^{3}$ DOFs in each element, and $(p+2)^{3}$ quadrature nodes.

The resolution at the wall is evaluated in wall units, i.e. $\Delta y^{+}=R e_{\tau}(\Delta y / h)$, where $\Delta y$ denotes the height of the first cell at the wall, and $R e_{\tau}=u_{\tau} h / \nu$ is the friction Reynolds number. The friction velocity is given by $u_{\tau}=\sqrt{\tau_{w} / \rho_{w}}$, being $\tau_{w}$ the wall-shear stress and $\rho_{w}$ the density at the wall. Note that in the DG approach, an estimate of $\Delta y^{+}$can be obtained by dividing the cell height by the number of DOFs within the element. For a polynomial approximation of degree $p$ we therefore define $y^{+}=\Delta y^{+} /(p+1) / 2$. For the simulations presented in Sec. 5.2 this leads to the following values : $y^{+}<2, \Delta x^{+}<34$, and $\Delta z^{+}<17$. These values are well within the recommendations given by Piomelli and Chasnov [53] for wall-resolving LES: $y^{+}<2, \Delta x^{+}=50-150$, and $\Delta z^{+} \approx 15-40$.

The normalised values of the time step used in the different computations are compiled in Table 2 . The CPU time per convective-time unit $t_{c}=12.8 \mathrm{~h} / \mathrm{u}_{b}$ required by each simulation is also provided in this table. Note that these times have been normalised by that of the cheapest simulation $D G-p 2$.

\subsection{Simulation results}

Figures 2 and 3 show a comparison between the profiles of mean velocity and velocity correlations obtained from the DG computations and the reference DNS data provided in [36]. It is important to bear in mind that the value of $u_{\tau}$ used to normalised the DG profiles is determined from the value of $R e_{\tau}$ yielded 

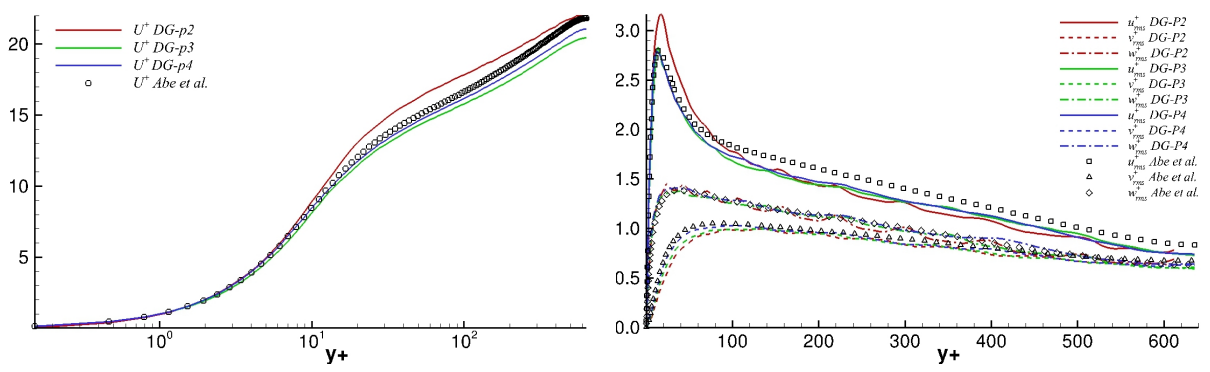

Figure 2: Profiles of mean velocity $U^{+}$(left) and rms of the velocity fluctuations $u_{r m s}^{+}, v_{r m s}^{+}$, $w_{r m s}^{+}$(right) for DG computations of the channel flow. Comparison against reference DNS data.

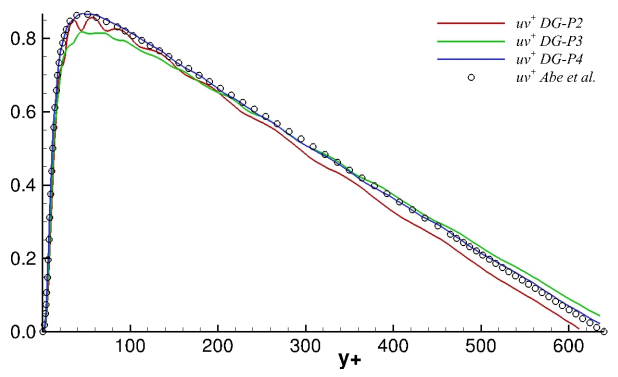

Figure 3: Profiles of cross correlations $-u v^{+}$for DG computations of the channel flow. Comparison against reference DNS data.

by the simulation (see Table 2). This allows us to assess the quality of our results in an objective manner.

We clearly see that the best accuracy is obtained when using a fifth-order approximation on a coarse mesh. These results highlight the benefit of increasing the polynomial order with respect to reducing the mesh size, as regards the accuracy of the solution. This point has also been demonstrated in previous work $[17,42,18]$.

Important discrepancies are found for the $D G-p 2$ computation with respect to the two other computations. These are probably due to the fact that the corresponding value of $R e_{\tau}$ is in this case significantly lower than the reference value of 640 , leading to an overestimation of the streamwise velocity. It is worth noting that the slight oscillations observed on the profiles of the root-meansquare (rms) of the velocity fluctuations are due to the discontinuous character of the DG solution. Indeed, we have found that the wavelength associated with these oscillations corresponds to the distance between elements.

As regards the solution to the passive scalar equation, only the $D G-p 4$ discretization has been considered. The reason is the very slow statistical convergence of this quantity, specially when using a compressible solver, which leads to a high computational cost. Figure 4 shows the profiles of the mean scalar and the correlations of the scalar and velocity fluctuations. These data have 

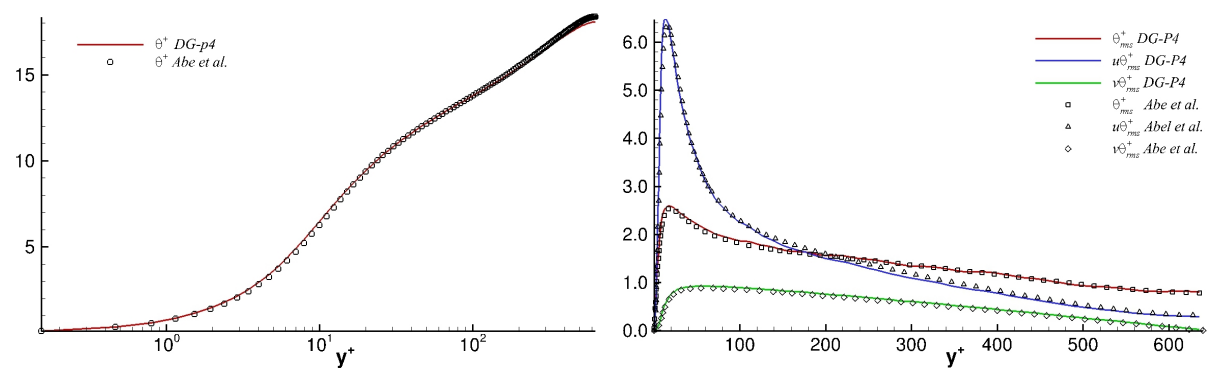

Figure 4: Profiles of mean scalar $\theta^{+}$(left) and rms $\theta_{r m s}^{+}$and correlations of the scalar and velocity fluctuations $u \theta^{+}$and $v \theta^{+}$(right) for $D G-p 4$ computation of the channel flow. Comparison against reference DNS data.

been normalised by $u_{\tau}$ and the friction temperature $\theta_{\tau}=Q_{w} / \rho_{w} c_{p} u_{\tau}$. We can observe the very good agreement found with the reference data. This is despite the great reduction in the number of DOFs with respect to the reference DNS.

\section{Turbulent flow over a 2D periodic hill}

In this section, we consider a fully developed turbulent flow over a $2 \mathrm{D}$ periodic hill. This test case was initially proposed in an ERCOFTAC/IAHR workshop in 1995. A detailed description of the geometrical configuration of the channel as well as of the flow conditions can be found on the ERCOFTAC QNET-CFD website [56].

This configuration represents the flow over periodically arranged hills. The flow presents separation from the curved surface, reattachment, and post reattachment recovery.

The dimensions of the channel are as follows : $L_{x}=9 h$ in the streamwise direction, $L_{y}=3.035 h$ in the vertical direction, and $L_{z}=4.5 h$ in the spanwise direction, where $h$ is the hill height at the crest. Periodicity of the flow is assumed in the streamwise and spanwise directions, and isothermal no-slip boundary conditions are imposed on the lower and upper walls. The flow is driven by a mean pressure gradient in the streamwise direction. This body force $\nabla_{x} p$ is implemented as a forcing term in the x-momentum and energy equations, as explained in Sec. 5.

The Mach number is set to $M_{0}=0.1$ so that the flow regime is quasiincompressible. The bulk Reynolds number $R e_{b}$ is defined based on the bulk velocity $u_{b}$ and on the hill height, $h$, namely,

$$
R e_{b}=\frac{u_{b} h}{\nu} \quad u_{b}=\frac{1}{2.035} \int_{h}^{3.035 h} u_{1}(y) d y
$$

Four different values of $R e_{b}$ have been considered: 2800, 10595, 19000, and 37000 . As already mentioned in Sec. 1 , the case at $R e_{b}=2800$ is considered as a DNS. For the higher values of $R e_{b}$ we employ an LES approach based 


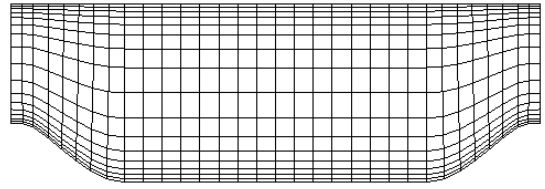

(a) Coarse mesh $32 \times 16 \times 16$

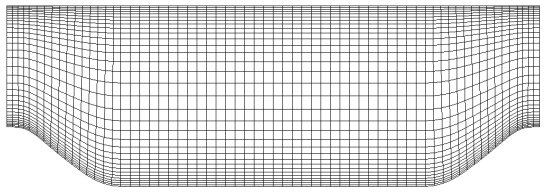

(b) Fine mesh $64 \times 32 \times 32$

Figure 5: Fourth-order meshes used in the DG simulations.

\begin{tabular}{llcc} 
& Grid resolution & \# Elements & Grid order \\
\hline LESOCC & - & $12.4 \mathrm{M}$ & 1 \\
\hline MGLET & $216 \times 168 \times 104$ & $3.77 \mathrm{M}$ & 1 \\
\hline Coarse mesh & $32 \times 16 \times 16$ & 8192 & 4 \\
\hline Fine mesh & $64 \times 32 \times 32$ & 65536 & 4 \\
\hline
\end{tabular}

Table 3: Grid resolutions used in the reference and DG computations.

on the WALE model [37]. An additional no-model LES computation of the $R e_{b}=10595$ configuration is also performed.

The following sections provide a summary of the computational parameters and the grids used in these simulations, as well as a summary of the main results obtained.

\subsection{Computational details}

Two levels of mesh refinement have been used in this study, shown in Fig. 5. A coarse fourth-order mesh composed of 8192 elements and a twice finer version of this mesh composed of 65536 elements. Both meshes were generated using the 3D finite element mesh generator Gmsh [57].The details of the high-order grids used for the DG simulations as well as those used in the reference FV computations in [38] (LESOCC code) and [39] (MGLET code) are provided in Table 3. The polynomial degree of the simulations is set to $p=3,4$ up to 5 for the DNS on the coarse grid, and $p=3$ and 4 for all other simulations.

As for the channel flow computations presented in the Sec. 5, the resolution at the wall is evaluated in terms of the normalised values $\Delta y^{+}=\Delta y u_{\tau} / \nu$. In this case, the friction velocity, $u_{\tau}$, is measured at the point of maximum wall-shear stress, which is located on the windward side of the hill as we will see in Sec. 6.2.1. Figure 6 shows the distribution of wall distances $y^{+}$and grid spacings $\left(\Delta x^{+}, \Delta z^{+}\right)$along the channel. The values of $y^{+}$at the point of maximum wall-shear stress are compiled in Table 4 . We observe that for the fifth-order DNS computation on the fine mesh a value of $y^{+}<1$ is found which is sufficient for a wall-resolved simulation. On the coarser meshes this value was close to 2 (not included in Table 4). For the higher-Reynolds-number cases the values of $y^{+}$are above the recommended value for a wall-resolving LES [53], although in line with the values reported in [39] for the MGLET simulations at 

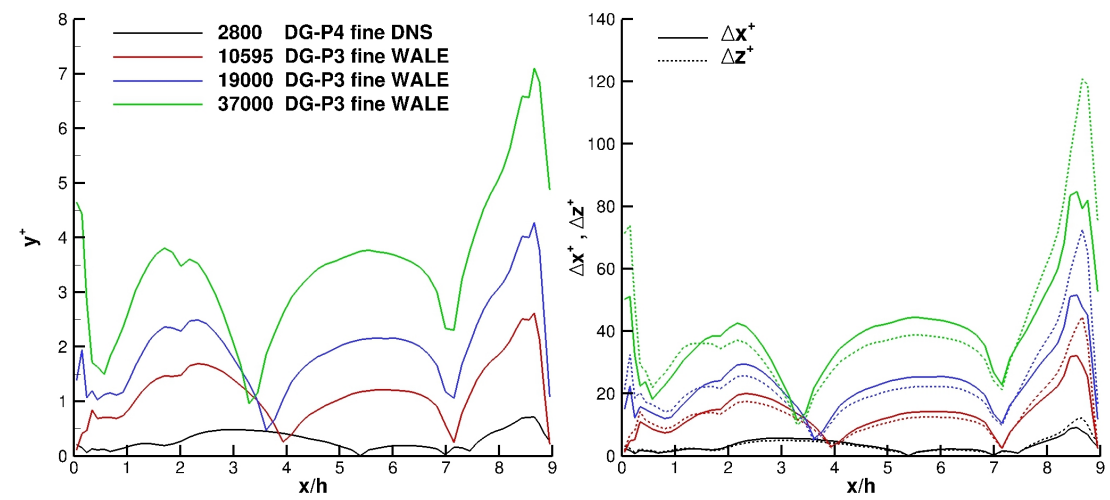

Figure 6: Streamwise distribution of $y^{+}$(right) and $\Delta x^{+}, \Delta z^{+}$(left) in the DG simulations.

\begin{tabular}{llccc}
$R e_{b}$ & LES approach & Simulation & \# DOFs & $y_{\max }^{+}$ \\
\hline 2800 & - & DG-p4 fine & $1.02 \mathrm{M}$ & 0.9 \\
\hline 10595 & no-model & DG-p3 fine & $4.19 \mathrm{M}$ & 2.9 \\
\hline 10595 & WALE & DG-p3 fine & $4.19 \mathrm{M}$ & 2.8 \\
\hline 19000 & WALE & DG-p3 fine & $4.19 \mathrm{M}$ & 4.5 \\
\hline 37000 & WALE & DG-p3 fine & $4.19 \mathrm{M}$ & 7.2 \\
\hline
\end{tabular}

Table 4: Wall resolution $y_{\max }^{+}$at point of maximum wall-shear stress for the DG simulations.

$R e_{b}=10595$ and $R e_{b}=37000$. As pointed out by the author, the distance over which this maximum wall stress is acting on the flow is very short $(\approx 0.5 h)$, and at all other positions the wall resolution can be considered as sufficient, as can be seen in Fig. 6.

The normalised time step used in the different computations is of the order of $10^{-4}$. It is worth noting that in this case the severe restriction on the time step arises from the convective time scale of the acoustic waves due to the low value of the Mach number $\left(M_{0}=0.1\right)$, and not from the viscous time scale associated with the penalty term in the SIP method.

The solution is advanced in time until a statistically steady state is reached. From this point, the flow statistics are gathered over a sufficient number of convective times $t_{c}=L_{x} / u_{b}$. The time-averaged data is also averaged in the spanwise direction to achieve more rapidly statistical convergence.

\subsection{Simulation results}

The results from the different DG simulations are analysed in detailed in the following sections. To this end, the profiles of mean streamwise and vertical velocity, as well as the turbulent stresses are compared against the available reference data at different stations along the streamwise direction. The locations 


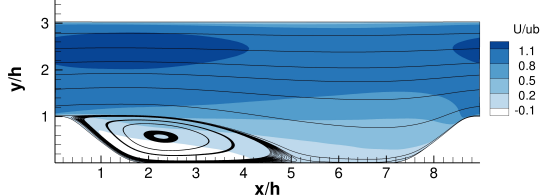

(a) $R e_{b}=2800$

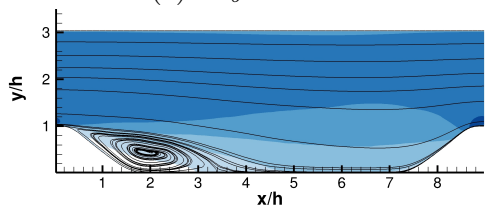

(b) $R e_{b}=19000$

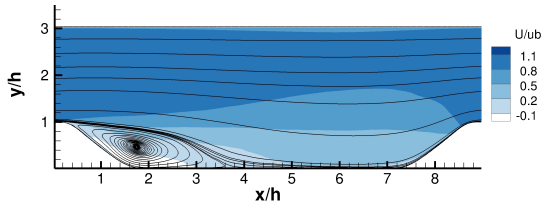

(b) $R e_{b}=10595$

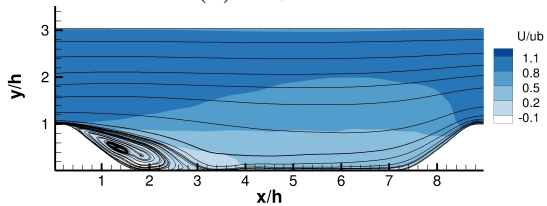

(c) $R e_{b}=37000$

Figure 7: Streamlines of the time-averaged streamwise velocity field $U / u_{b}$ from DG simulations (see Table 4).

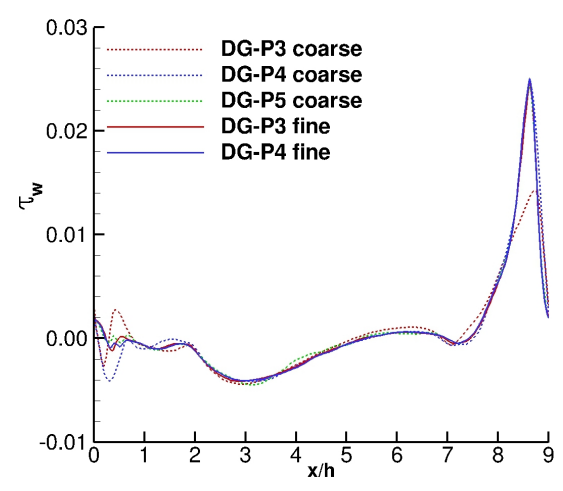

(a) $R e_{b}=2800$

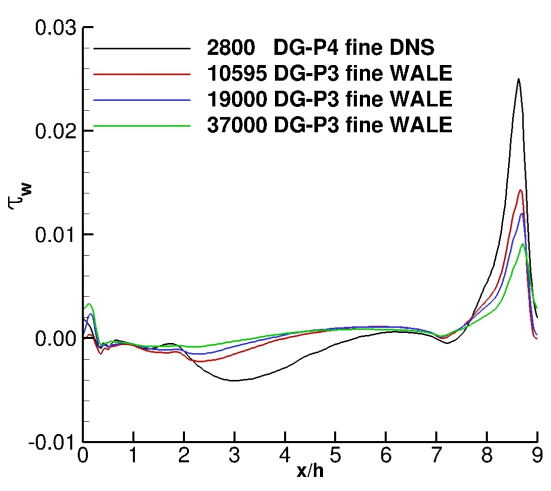

(b) $R e_{b}=2800$ up to $R e_{b}=37000$

Figure 8: Profiles of wall-shear stress at bottom wall for the DG simulations.

inspected correspond to $x / h=0.05,0.5,2,4,6$, and 8 . We pay particular attention to stations $x / h=0.5,2,4$, and 6 . The first $x / h=0.5$ corresponds to a position located right after the separation region and intersects the strong shear layer generating from the detachment point. The second station $x / h=2$ is located in the middle of the recirculation region. Station $x / h=4$ is placed at the end of the recirculation bubble (close to the reattachment point for the lower Reynolds numbers) and $x / h=6$ is located downstream the recirculation zone in the reattached flow.

The DG solutions are compared to the CFD data provided by Breuer et al. $\left(R e_{b}=2800\right.$ and $\left.R e_{b}=10595\right)$ using the second-order FV incompressible solver LESOCC on a curvilinear grid [38] and by Manhart et al. $\left(R e_{b}=37000\right)$ using the second-order FV incompressible solver MGLET on a Cartesian mesh [39]. All LES results are also compared to the experimental data provided by 


\begin{tabular}{lcccc} 
Simulation & \#DOFs & $\Delta t\left(u_{b} / h\right) / 10^{-4}$ & \#Cores & CPU time $/ t c$ \\
\hline LESOCC FV-p1 & $13.1 \mathrm{M}$ & 20 & - & - \\
\hline DG-p3 Coarse & $0.52 \mathrm{M}$ & 2.5 & 264 & 1.0 \\
\hline DG-p4 Coarse & $1.02 \mathrm{M}$ & 2.5 & 264 & 2.8 \\
\hline DG-p5 Coarse & $1.77 \mathrm{M}$ & 1.7 & 264 & 13.1 \\
\hline DG-p3 Fine & $4.19 \mathrm{M}$ & 1.8 & 2208 & 10.8 \\
\hline DG-p4 Fine & $8.19 \mathrm{M}$ & 1.4 & 2208 & 39.9 \\
\hline
\end{tabular}

Table 5: Computational details of DG-DNS computations at $R e_{b}=2800$.

Rapp [40].

6.2.1. Qualitative comparison of DG solutions at $R_{b}=2800$ up to 37000

The streamlines of the time-averaged velocity field for $R e_{b}=2800$ up to 37000 are depicted in Fig. 7. As expected, the reattachment point moves upstream with increasing Reynolds number, reducing the size of the recirculation bubble. The position of the reattachment point at the lowest Reynolds number is $x_{R} / h \approx$ 5.4 which is in agreement with the value reported in [38]. For $R e_{b}=10595$ the reattachment point is located at $x_{R} / h \approx 3.9$, which is slightly different from the experimental value $\left(x_{R} / h \approx 4.21\right)$ [55] and from the reference LESOCC simulation $\left(x_{R} / h \approx 4.69\right)$. Finally, for the highest Reynolds number $R e_{b}=37000$ the reattachment point is located at $x_{R} / h \approx 3.2$ not far from the value of $x_{R} / h \approx 3.68$ reported by Chaouat and Schiestel [54] in their hybrid RANS/LES simulation on a grid composed of $1 \mathrm{M}$ points.

The profiles of time-averaged wall-shear stress at the bottom wall for $\operatorname{Re}_{b}=2800$ are depicted in Fig. 8(a). We observe that all discretizations, except for the $D G-p 3$ simulation on the coarse mesh, are able to reproduce the peak of maximum wall-shear stress. The strong oscillations near the separation point exhibited by the $D G-p 3$ and $D G-p 4$ coarse-mesh simulations are clearly due to a lack of resolution in this area. Indeed, this oscillating behaviour disappears when the polynomial degree is increased. This is shown by the results obtained from the $D G-p 5$ coarse-mesh simulation, which closely match the data obtained from the best resolved simulations for a reduced number of degrees of freedom (1.77 $\mathrm{M}$ in the $D G-p 5$ with respect to $4.19 \mathrm{M}$ and $8.19 \mathrm{M}$ in the $D G-p 3$ and $D G-p 4$ fine-mesh simulations, respectively). This outcome emphasises the greater advantage of using local $h p$-adaptation for flow configurations featuring highly localised phenomena.

In Fig. 8(b) we compare the profiles of wall-shear stress for all four Reynolds numbers. The trends are in agreement with the results reported in [38].

\section{3. $D N S$ results at $R e_{b}=2800$}

Table 5 summarises the five DNS simulations performed at $R e_{b}=2800$. The $\mathrm{CPU}$ time required to advance one convective time is also shown in this table. 
Note, that this cost is normalised by that of the cheapest DNS computation, i.e. $D G-p 3$ on the coarse mesh.

Figures 10 and 11 show the profiles of averaged velocity and turbulent stresses obtained from the DG-DNS at the different levels of resolution considered (see Table 5). They are compared to the DNS data from the LESOCC code. It can be observed that all DG simulations are able to reproduce the main features of the flow (separation, recirculation and reattachment). Naturally, the best match with the reference DNS is obtained for the $D G-p 4$ simulation on the fine grid (8.19 M DOFs), as we reach numerical convergence in terms of number of degrees of freedom.

However, if we look carefully at the solution from the $D G-p 5$ coarse-mesh simulation we observe that the match with the $D G-p 4$ fine-mesh simulation and the reference data is remarkably good. This is taking into account the great reduction in the number of DOFs with respect to the two other simulations (8.19 $\mathrm{M}$ and $13.1 \mathrm{M}$, respectively). It is also worth noting that the computational cost of the $D G-p 5$ is significantly lower than that of the $D G-p 4$ simulation and only slightly higher than that of the $D G-p 3$ simulation on the fine mesh (see Table 5).

As already discussed in the previous section, the $D G-p 3$ and $D G-p 4$ coarse-mesh simulations exhibit an oscillatory behaviour in the vicinity of the separation point (see Fig. 10). These oscillations disappear when the polynomial order is increased to $p=5$. We can also observe the presence of spurious overshoots in the profiles of $\left\langle u^{\prime} u^{\prime}\right\rangle$ at practically all stations. The locations of these peaks actually coincide with the grid element interfaces and are a consequence of the discontinuous character of the DG solutions at this coarse resolution. These jumps are smoothed out when the polynomial degree is increased, as can be observed from the $\left\langle u^{\prime} u^{\prime}\right\rangle$ profiles in the $D G-p 5$ simulation.

We can conclude from this analysis that an optimal performance of the DG method could be obtained by appropriate local $h p$-adaptation. This would allow us to greatly reduce the cost of the simulation while keeping the same level of accuracy as that provided by uniform $h$ and/or $p$-refinement.

\subsection{LES results at $R_{b}=10595$}

Figures 12 and 13 show the profiles of mean velocity and turbulent stresses obtained from the $D G-p 3$ fine-mesh simulations using the no-model and the WALE approaches. They are compared to the LES data produced by the LESOCC code as well as to the experimental data from Rapp [40].

It can be seen from these plots that at this relatively low Reynolds number the results yielded by the no-model and the WALE approaches are not fundamentally different. The WALE approach, however, seems to provide slightly better results in the region close behind the separation point $(x=0.5 h)$. This is specially the case for the mean vertical velocity profile as well as for the turbulent stresses, for which the no-model simulation presents a more marked overshoot near the wall. The profiles obtained using the WALE approach appear to be closer to the reference data. These comments also hold for the area located 


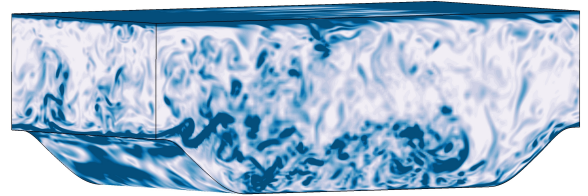

(a) $R e_{b}=2800$

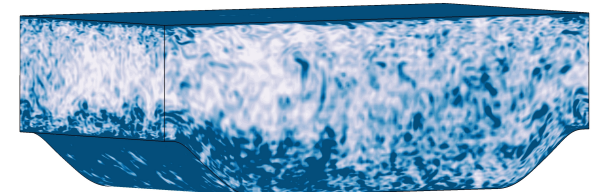

(b) $R e_{b}=10595$

Figure 9: Instantaneous field of vorticity magnitude at $R e_{b}=2800$ and 10595 .

in the middle of the separation zone (corresponding to $x=2 h$ ), although the differences become gradually less significant as we exit the recirculation zone $(x=4 h)$. Finally, in the area well behind the recirculation bubble $(x=6 h)$ both simulations provide similar results. However, the WALE simulation appears to represent with higher fidelity the mean vertical velocity profile which is closer to the experiment, though significantly far from the simulation.

It is also worth noting the smoothing effect that the addition of the subgrid model has on $\left\langle u^{\prime} u^{\prime}\right\rangle$. Indeed, at all stations, we observe that the unphysical peaks generated by the DG method at the element interfaces are considerably damped when the WALE approach is used.

Figures 9(a) and (b) show the field of instantaneous vorticity magnitude displayed by the DG solutions at $R e_{b}=2800$ and 10595 , respectively. We can see on these plots the finer structures present in the flow at this higher $R e_{b}$.

\subsection{LES results at $R e_{b}=19000$}

Figures 14 and 15 show the profiles of mean velocity and turbulent stresses obtained from the $D G-p 3$ fine-mesh simulation using the WALE approach. They are compared to the experimental data from Rapp [40].

In view of the results from the previous section, only the WALE approach has been considered at this higher Reynolds number. In particular, due to the fact that the raw DG scheme did not appear to provide a sufficient level of dissipation to achieve a stable simulation. This was also the case for the $R e_{b}=37000$ simulation.

An overall good agreement is found between the results from this simulation and the experimental data. The main differences are found in the vicinity of the separation point. At this location the profiles of $\left\langle u^{\prime} v^{\prime}\right\rangle$ from the DG simulation present a strong overshoot near the wall. This result might suggest a need to improve the resolution in this area. In the recirculation region $(x=$ $2 h$ ) however, the numerical solution is in relatively good agreement with the experimental data. Right after the recirculation bubble $(x=4 h$ and $x=6 h)$ the match between the mean vertical velocity profiles deteriorates, while at the final stations $(x=8 h)$ the agreement between the DG profiles and the experiment improves considerably.

It is difficult discern, however, whether the discrepancies observed at some stations in the channel are due to numerical errors, or to measurement uncertainties during the experiment. Further DG simulations at this higher Reynolds 
number, ideally using a locally $h p$-adapted grid, could help understand the nature of these differences.

\section{6. $L E S$ at $\operatorname{Re}_{b}=37000$}

Figures 16 and 17 show the profiles of mean velocity and turbulent stresses obtained from the $D G-p 3$ fine-mesh simulation using the WALE approach. They are compared to the LES data produced by the MGLET code [39] as well as to the experimental data from Rapp [40].

An overall good agreement is found between the mean streamwise velocity profiles and the reference data. Some discrepancies between the experimental data and the DG solution are found however for the mean vertical velocity profiles, which appear in general to be closer to the reference CFD data. The match between the turbulent stresses $\left\langle u^{\prime} u^{\prime}\right\rangle$ and $\left\langle u^{\prime} v^{\prime}\right\rangle$ from the DG simulation and the experiment is relatively good. Note that the overshoot that appears near the bottom wall at $x=0.5 h$ in both profiles is also observed in the MGLET profiles as well as in the numerical results reported by Chaouat and Schiestel in [54].

\section{Conclusions}

The accuracy of a modal DG approach to simulate wall-bounded turbulent flows for a range of Reynolds numbers has been assessed in this work.

Firstly, under-resolved DNS computations of a plane channel flow configuration with turbulent heat transfer at $R e_{\tau}=640$ have been carried out. The DG simulations involve four times less DOFs in each space direction than the reference DNS simulation of Abe et al. [49]. In the absence of a subgrid model, a simple de-aliasing of the solution via overintegration is sufficient to achieve a stable simulation at this relatively low Reynolds number. The $h p$-convergence analyses clearly demonstrate the greater advantage of using high-order approximations on very coarse meshes, as opposed to mesh refinement in combination with low-order schemes. We actually find the best match with the reference DNS data for the fifth-order simulation on the coarsest mesh.

In a second stage, the ability of the DG method to capture the Reynoldsnumber dependent features in a detached flow configuration has been investigated for Reynolds numbers ranging from $R e_{b}=2800$ up to 37000 . As for the channel flow configuration, $h p$-convergence studies have been carried out for the DNS at $R e_{b}=2800$. Once more, the outcome from this analysis demonstrates the superior performance of increasing the polynomial order as compared to refining the mesh, specially in the area close to separation. A very good agreement with the reference simulation of Breuer et al. [38] is actually obtained by considering a sixth-order discretization $(p=5)$ on a very coarse mesh, involving only $1.77 \mathrm{M}$ DOFs with respect to the $13.1 \mathrm{M}$ DOFs used in the reference FV computation. This result emphasises the great advantage of using local $h p$ adaptation for flow configurations featuring highly localised phenomena, as is the case here. 
For $R e_{b}=10595,19000$, and 37000 fourth-order LES $(p=3)$ based on the WALE approach have been carried out on a grid composed of 65536 elements, which leads to $4.19 \mathrm{M}$ DOFs. All simulations are able to capture the main features of the flow. In particular, a good agreement with the reference data is found for $R e_{b}=10595$ and $R e_{b}=19000$. The discrepancies found for $R e_{b}=37000$ between the DG simulation and the experiment are in line with those reported in [39] using the MGLET FV code on a mesh composed of $4.1 \mathrm{M}$ points and in [54] using a hybrid RANS/LES approach on a $1 \mathrm{M}$ point grid. An additional no-model LES simulation has been performed at $R e_{b}=10595$. The solutions from the no-model and the WALE approaches do not appear to be fundamentally different. The WALE approach, however, seems to provide slightly more accurate results, specially in the vicinity of the separation point and in the

recirculation area. The smoothing effect that the addition of the subgrid model has on the $\left\langle u^{\prime} u^{\prime}\right\rangle$ profiles has also been highlighted. Indeed, at all stations, we observe that the unphysical peaks generated by the interface jumps of the DG solution are considerably damped by the use of a subgrid model. Note that for $R e_{b}=19000$ and 37000 the no-model approach did not yield stable simulations. This is a manifestation of the very low dissipation level provided by the DG scheme, and the need to use subgrid-scale modelling when we move towards higher Reynolds numbers. We see from these results that the combined used of local $h p$-adaptation with a subgrid-scale modelling approach could fully exploit the benefits of DG methods for industrial LES. Furthermore, in order to reduce the computational cost associated with the use of high polynomial degrees it would be advantageous to use a hybrid MPI/openMP approach, instead of the pure MPI approach used in this work. This is the subject of current research in the framework of the Aghora project.

\section{Acknowledgments}

This research was partly funded by the European H2020 TILDA project, with additional support from Safran Group. The authors would like to thank Dr. E. Martin for his help concerning parallel computing issues. We also thank Dr. J.-B. Chapelier for his help with the plane channel configuration during his postdoctoral work at Onera. This work was performed using HPC resources from GENCI (Grant 2015 - c20152a7441).

\section{References}

[1] Orszag S. A. and Patterson G. S., Numerical simulation of threedimensional homogeneous isotropic turbulence, Phys. Rev. Lett. 28 (1972) pp. 76-79.

[2] Sagaut P., Large Eddy Simulation for Incompressible Flows: An Introduction, Springer, 2006.

[3] Garnier E., Adams N., and Sagaut P., Large Eddy Simulation for Compressible Flows, Springer, Berlin (2009) 
[4] P. Lesaint and P.-A. Raviart, On a finite element method for solving the neutron transport equation, in Mathematical Aspects of Finite Elements in Partial Differential Equations, de Boor ed., Academic Press, New York, 1974, pp. 89-123.

[5] W. H. Reed and T. R. Hill, Triangular mesh methods for the neutron transport equation, Technical Report LA-UR-73-479, Los Alamos Scientific Laboratory, NM, 1973.

[6] B. Cockburn and C. W. Shu, TVB Runge-Kutta local projection discontinuous Galerkin finite element method for scalar conservation laws II: general framework, Math. Comp. 52 (1989), pp. 411-435.

[7] B. Cockburn and C. W. Shu, Runge-Kutta discontinuous Galerkin methods for convection-dominated problems, J. Sci. Computing 16 (2001), pp. 173261.

[8] R. Biswas, K. Devine and J.E. Flaherty, Parallel adaptive finite element methods for conservation laws, Appl. Numer. Math. 14 (1994) pp. 255284.

[9] Kroll N., Bieler H., Deconinck H., Couaillier V., van der Ven H., and Sørensen K., ADIGMA - A European Initiative on the Development of Adaptive Higher-Order Variational Methods for Aerospace Applications, Notes on Numerical Fluid Mechanics and Multidisciplinary Design, 113 (Springer, 2010).

[10] Kroll N., Hirsch C., Bassi F., Johnston C., Hillewaert, K. IDIHOM: Industrialization of High-Order Methods - A Top-Down Approach, Notes on Numerical Fluid Mechanics and Multidisciplinary Design Series, 128 (Springer, 2015).

[11] Collis S., Discontinuous Galerkin methods for turbulence simulation, in: Center for Turbulence Research, Proceedings of the Summer Program, 2002 pp. $155-167$.

[12] Abbas Q. and Nordström, Weak versus strong no-slip boundary conditions for the NavierStokes equations, Eng. Appl. Comput. Fluid Mech. 4 (2010) pp. 29-38.

[13] Nordström J., Eriksson S., and Eliasson P., Weak and strong wall boundary procedures and convergence to steady-state of the NavierStokes equations, J. Comput. Phys. 231 (2012) pp. 4867-4884.

[14] P. Houston and E. Süli, $h p$-adaptive discontinuous Galerkin finite/ element methods for first-order hyperbolic problems. SIAM J. Sci. Comput. 23 (2001), pp. 1226-1252. 
[15] Wei L. and Pollard A., Direct numerical simulation of compressible turbulent channel flows using the discontinuous Galerkin method, Comput. Fluids 47 (1) (2011) pp. 85-100.

[16] Wei L. and Pollard A., Interactions among pressure, density, vorticity and their gradients in compressible turbulent channel flows, J. Fluid Mech. 673 (2011) pp. 1-18.

[17] Chapelier J.-B., de la Llave Plata M., and Renac F., Inviscid and viscous simulations of the Taylor-green vortex flow using a modal discontinuous Galerkin approach, 42nd AIAA Fluid Dynamics Conference and Exhibit, 2012.

[18] Chapelier J.-B., de la Llave Plata M., Renac F., and Lamballais E., Evaluation of a high-order DG method for the DNS of turbulent flows, Comp. Fluids, 95 (2014) pp. 206-226.

[19] Diosady L. and Murman S. M., 2014 DNS of Flows over Periodic Hills using a Discontinuous Galerkin Spectral-Element Method. AIAA Paper $2014-2784$.

[20] Garai A., Diosady L.T., Murman S.M., and Madavan N., DNS of Flow in a Low-Pressure Turbine Cascade with Elevated Inflow Turbulence Using a Discontinuous-Galerkin Spectral-Element Method, in Proceedings of ASME Turbo Expo 2016, no. GT2016-56700, 2016.

[21] Bassi F., Botti L., Colombo A., Crivellini A., Ghidoni A., and Massa F., On the development of an implicit high-order Discontinuous Galerkin method for DNS and implicit LES of turbulent flows, European J. Mech. - B/Fluids, 55 (2) (2016) pp. 367-379.

[22] Gassner G. J. and Beck A. D., On the accuracy of high-order discretizations for underresolved turbulence simulations, Theor. Comput. Fluid Dyn. 27 (3-4) (2013) pp. 221237.

[23] Uranga A., Persson P., Drela M. and Peraire J., Implicit Large-Eddy Simulation of transition to turbulence at low Reynolds numbers using a discontinuous Galerkin method, Int. J. Numer. Meth. Eng. 87 (1-5) (2011) pp. 232-261.

[24] Carton de Wiart C., Hillewaert K., Bricteux L., and Winckelmans G., Implicit LES of free and wall-bounded turbulent flows based on the discontinuous Galerkin/symmetric interior penalty method, Int. J. Numer. Meth. Fluids 78 (6) (2015) pp. 335-354.

[25] Beck A. D., Bolemann T., Flad D., Frank H., Gassner G., Hindenlang F., and Munz C.-D., High-order discontinuous Galerkin spectral element methods for transitional and turbulent flow simulations, Int. J. Numer. Meth. Fluids 76 (8) (2014) pp. 522-548. 
[26] Beck, A.D., Bolemann, T., Flad, D., Frank, H., Gassner, G., Hindenlang, F., Munz, C.-D., High-order discontinuous Galerkin spectral element methods for transitional and turbulent flow simulations, Int. J. Numer. Methods Fluids, 76 (2014) pp. 522-548.

[27] Bolemann T., Beck A., Flad D., Frank H., Mayer V., and Munz C.-D., High-order discontinuous Galerkin schemes for Large-Eddy simulations of moderate Reynolds number flows, in: IDIHOM: Industrialization of HighOrder Methods-A Top-Down Approach, Springer, 2015, pp. 435-456.

[28] Flad D., Beck A., Munz C.-D., Simulation of underresolved turbulent flows by adaptive filtering using the high order discontinuous Galerkin spectral element method, J. Comput. Phys. 313 (2016) pp. 1-12.

[29] Kirby, R.M. and Karniadakis, G.E., De-aliasing on non-uniform grids: algorithms and applications, J. Comput. Phys. 191 (2003) pp. 249-264.

[30] Mengaldo G., De Grazia D., Moxey D., Vincent P.E., and Sherwin S.J., De-aliasing techniques for high-order spectral element methods on regular and irregular grids, J. Comput. Phys. 299 (2015) pp. 56-81.

[31] van der Bos F. and Geurts B., Computational error-analysis of a discontinuous Galerkin discretization applied to Large-Eddy Simulation of homogeneous turbulence, Comput. Meth. Appl. Mech. Eng. 199 (2010) pp. 903915.

[32] Marek M., Tyliszczak A., and Boguslawski A., Large-eddy simulation of incompressible free round jet with discontinuous Galerkin method, Int. J. Numer. Meth. Fluids 79 (4) (2015) pp. 164-182.

[33] Abbà A., Bonaventura L., Ninia M., and Restellic M., Dynamic models for Large-Eddy Simulation of compressible flows with a high-order DG method, Comput. Fluids 122 (2015) pp. 209-222.

[34] Chapelier J.-B., de la Llave Plata M., and Lamballais E., Development of a multiscale LES model in the context of a modal discontinuous Galerkin method, Comput. Meth. Appl. Mech. Eng. 307 (2016) pp. 275-299.

[35] Beck A., Flad D., Tonhäuser C., Gassner G., and Munz C.-D., On the Influence of Polynomial De-aliasing on Subgrid Scale Models, Flow Turbul. Combust. (2016) pp. 1-37.

[36] DNS database of Kawamura lab: http://murasun.me.noda.tus.ac.jp/turbulence/menu.html

[37] Nicoud F., Ducros F., Subgrid-Scale Stress Modelling Based on the Square of the Velocity Gradient Tensor, Flow Turbul. Combust. 62 (1999) pp. 183200. 
[38] Breuer M., Peller N., Rapp Ch., Manhart M., Flow over periodic hills Numerical and experimental study in a wide range of Re, Comput. Fluids 38 (2009), pp. 433-457.

[39] Manhart M., Rapp Ch., Peller N., Breuer M., Aybay O., Denev J., Falconi C.J., Assessment of eddy resolving techniques for the flow over periodically arranged hills up to $R e=37,000$, In: Salvetti M.V., Geurts B., Meyers J., Sagaut P. (eds) Quality and Reliability of LES II, ERCOFTAC Series, 16 pp. 361-37 (Springer 2011)

[40] Rapp Ch., Experimentelle Studie der turbulenten Strömung über periodische Hügel, PhD Thesis 2009, Technische Universität München, Germany.

[41] Lesieur M., Métais O., Comte P., Large-eddy simulations of turbulence, Cambridge University Press, Cambridge 2005.

[42] Chapelier J.-B., Développement et évaluation de la méthode de Galerkin discontinue pour la simulation des grandes échelles des écoulements turbulents, PhD Thesis 2013, Université de Bordeaux.

[43] F. Ducros, P. Comte, and M. Lesieur, LES of a spatially growing boundary layer over an adiabatic flat plate at low Mach number, Int. J. Heat Fluid Flow, 16 (1995) pp 341-348.

[44] Renac F., de la Llave Plata M., Martin E., Chapelier J.-B., Couaillier C., Aghora: A High-Order DG Solver for Turbulent Flow Simulations, IDIHOM: Industrialisation of High-Order Methods - A Top Down Approach, Notes on Numerical Fluid Mechanics and Multidisciplinary Design, 128 (Springer 2015) pp 315-335.

[45] Bassi F., Botti L., Colombo A., Di Pietro D.A., Tesini P., On the flexibility of agglomeration based physical space DG discretizations, J. Comput. Phys. 231 (2012) pp. 45-65.

[46] Gottlieb S., Shu C.W., Tadmor E., Strong stability-preserving high-order time discretization methods, SIAM review, 43 (2001) pp. 89-112.

[47] Arnold D.N., Brezzi F., Cockburn B., Marini L. D., Unified Analysis of Discontinuous Galerkin Methods for Elliptic Problems, SIAM J. Numer. Anal. 39 (2001) pp. 1749-1779.

[48] R. Hartmann and P. Houston, An optimal order interior penalty discontinuous Galerkin discretization of the compressible Navier-Stokes equations J. Comput. Phys. 227 (2008) pp. 9670-9685.

[49] Abe H., Kawamura H., and Matsuo Y., Surface heat-flux fluctuations in a turbulent channel flow up to $R e_{\tau}=1020$ with $\operatorname{Pr}=0.025$ and $0.7,1$ Int. J. Heat Fluid Flow 25 (2004) 404-419. 
[50] Deschamps V., Simulation numérique de la turbulence inhomogène incompressible dans un écoulement de canal plan, Note technique ONERA, 1988.

[51] Lenormand E., Contribution à la simulation des grandes échelles d'écoulements turbulents compressibles en milieux confinés, PhD Thesis, Université de Paris VI, 1999.

[52] Lenormand E., Sagaut P., and Ta Phuoc L., Large-eddy simulation of compressible channel flow at moderate Reynolds number, Int. J. Numer. Methods Fluids, 32 (2000), pp. 369-406

[53] U. Piomelli and J.R. Chasnov, Large-Eddy Simulations: Theory and Applications, In Turbulence and Transition Modelling, ERCOFTAC Series, 2 pp 269-336 (Springer 1996).

[54] B. Chaouat and R. Schiestel, Hybrid RANS-LES Simulations of the Turbulent Flow over Periodic Hills at High Reynolds Number, Comput. \& Fluids, 84 (2013) pp. 279-300.

[55] Rapp C.R., Manhart C., Flow over periodic hills - an experimental study, Exp. Fluids 51 (2011) pp. 247-269.

[56] The ERCOFTAC QNET-CFD Wiki Home Page: qnet-ercoftac.cfms.org.uk

[57] C. Geuzaine and J.-F. Remacle. Gmsh: a three-dimensional finite element mesh generator with built-in pre- and post-processing facilities, Int. J. Numer. Meth. Eng. 79 (2009) pp. 1309-1331. 

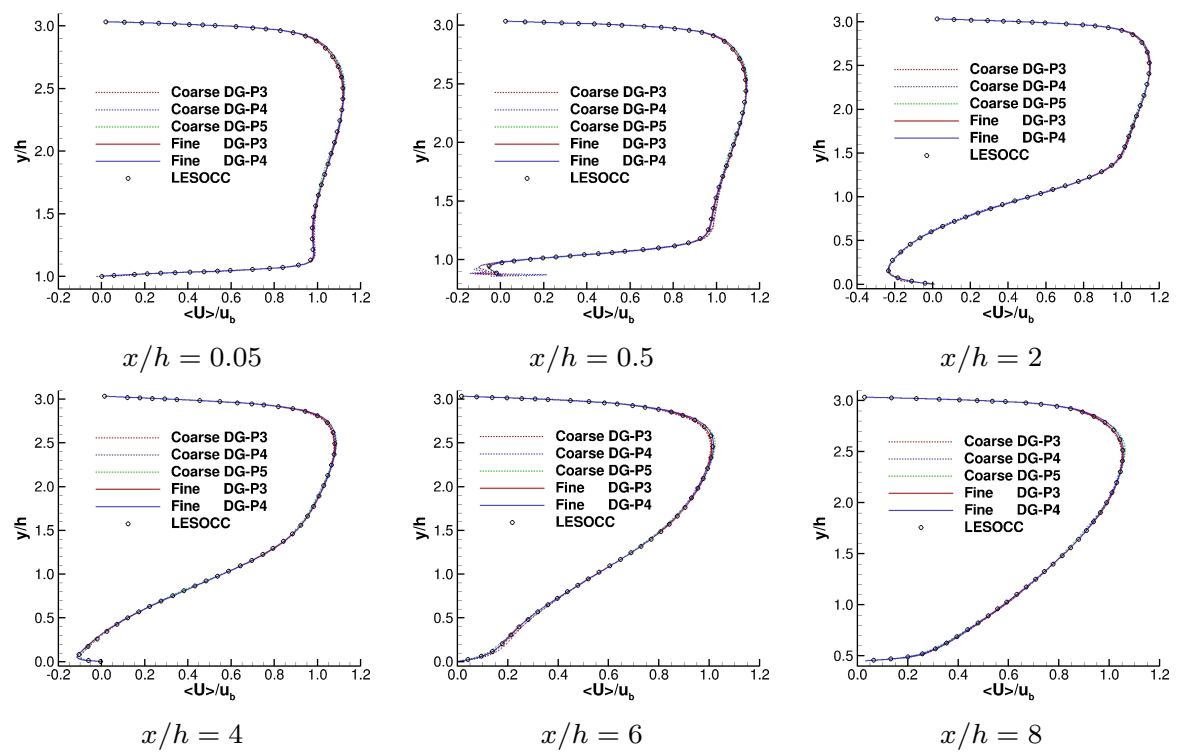

(a) Mean streamwise velocity $U / u_{b}$ profiles
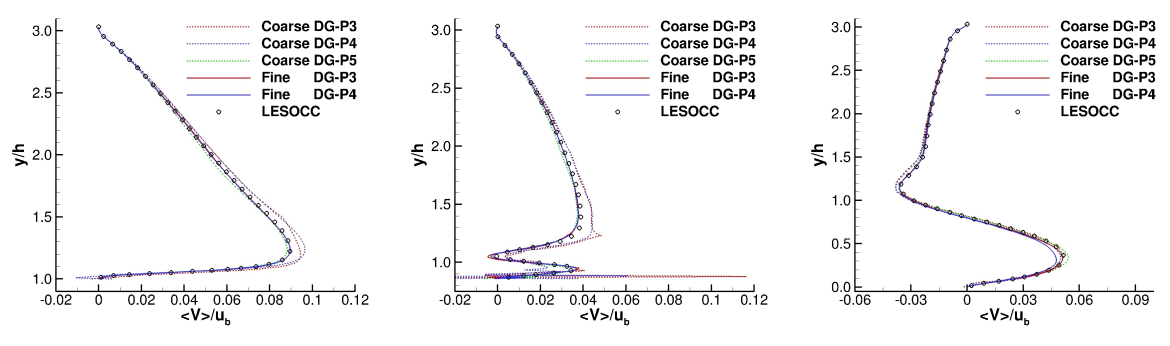

$x / h=0.05$

$x / h=0.5$
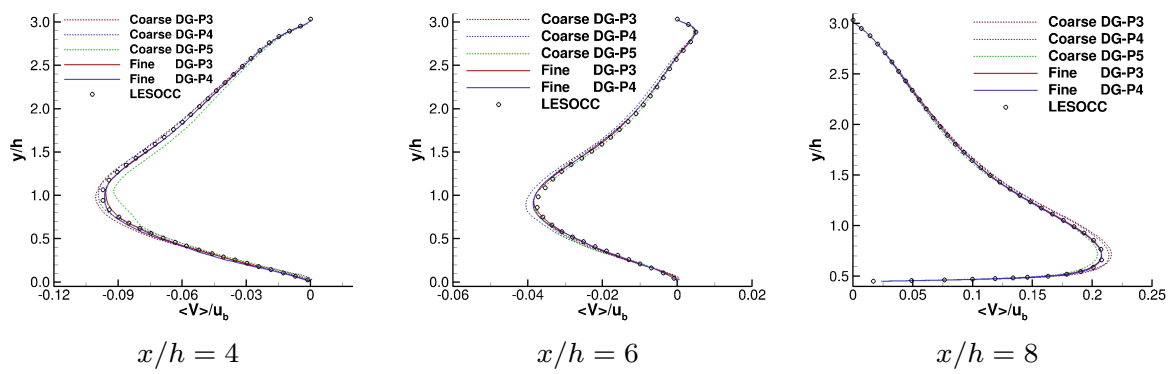

(b) Mean vertical velocity $V / u_{b}$ profiles

Figure 10: DNS at $R e_{b}=2800$. Comparison of DG simulations with reference data. $\circ$ : LESOCC $O(2) ;--$ : $D G-p 3$ coarse; - - : $D G-p 4$ coarse; - - : $D G-p 5$ coarse; - : $D G-p 3$ fine; - : $D G-p 4$ fine. 

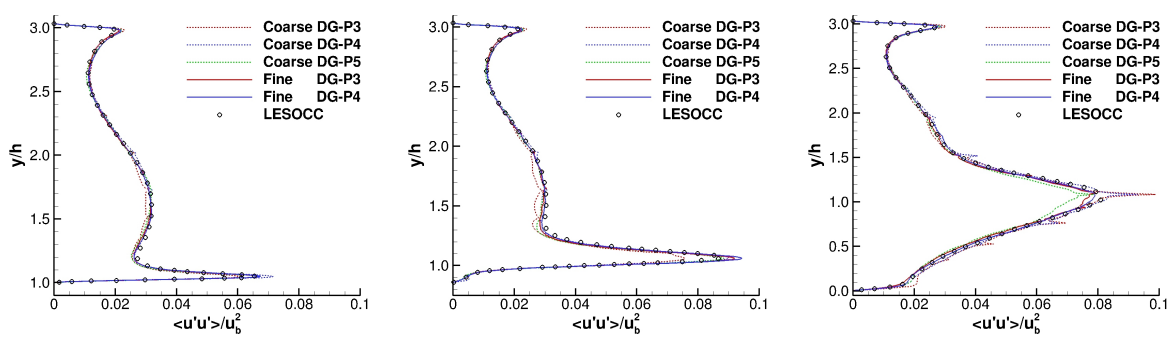

$x / h=0.05$
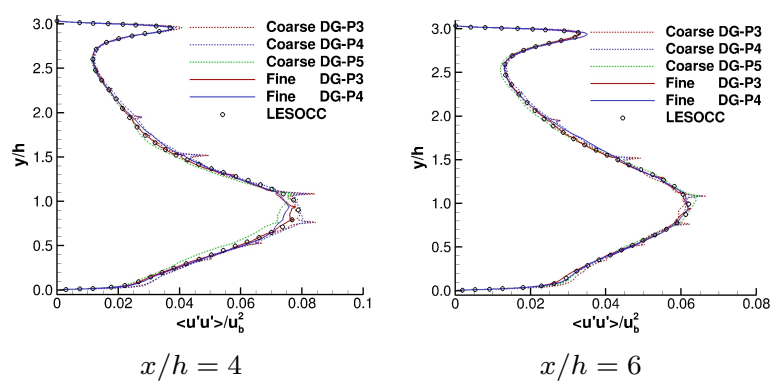

$x / h=2$

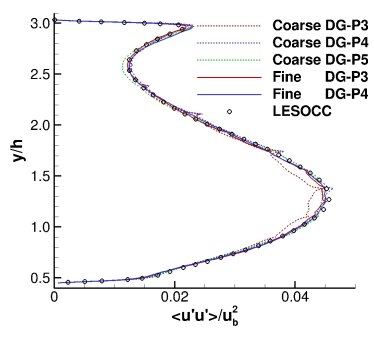

(a) Reynolds stress $\left\langle u^{\prime} u^{\prime}\right\rangle / u_{b}^{2}$ profiles
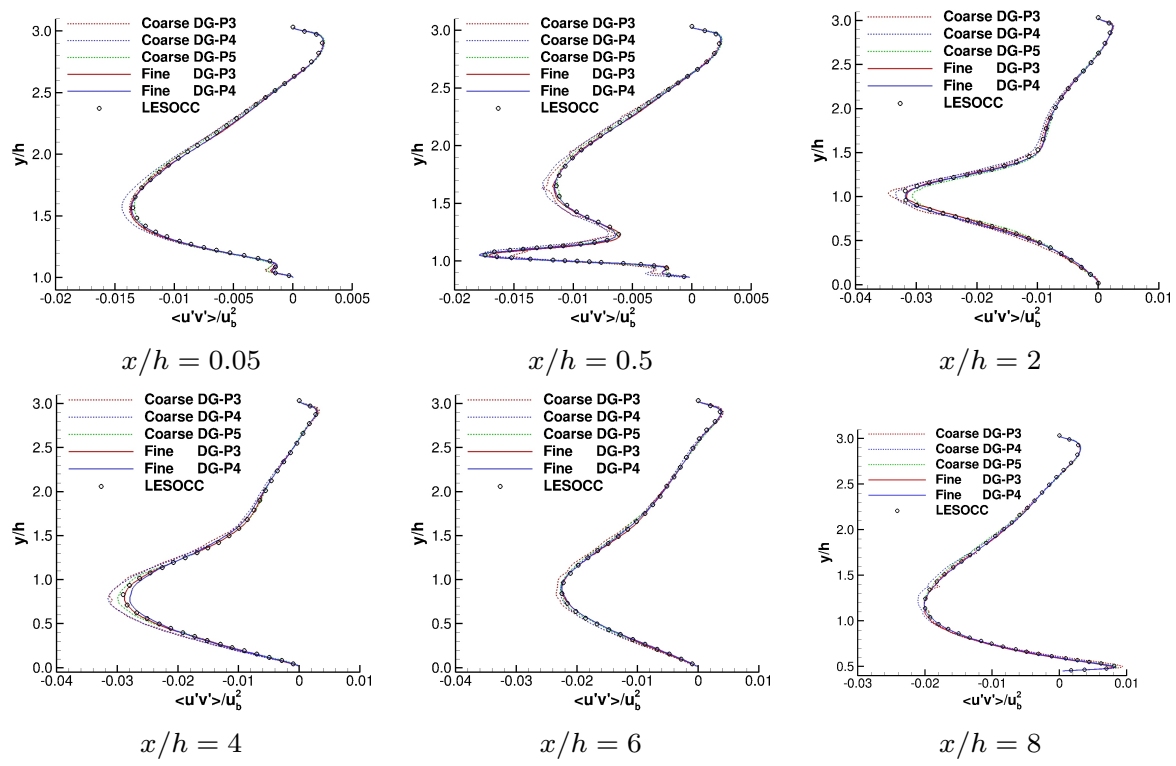

(b) Shear stress $\left\langle u^{\prime} v^{\prime}\right\rangle / u_{b}^{2}$ profiles

Figure 11: DNS at $R e_{b}=2800$. Comparison of DG simulations with reference data. $\circ$ : LESOCC $O(2) ;--$ : $D G-p 3$ coarse; - - : $D G-p 4$ coarse; - - : $D G-p 5$ coarse; - : $D G-p 3$ fine; - : $D G-p 4$ fine. 

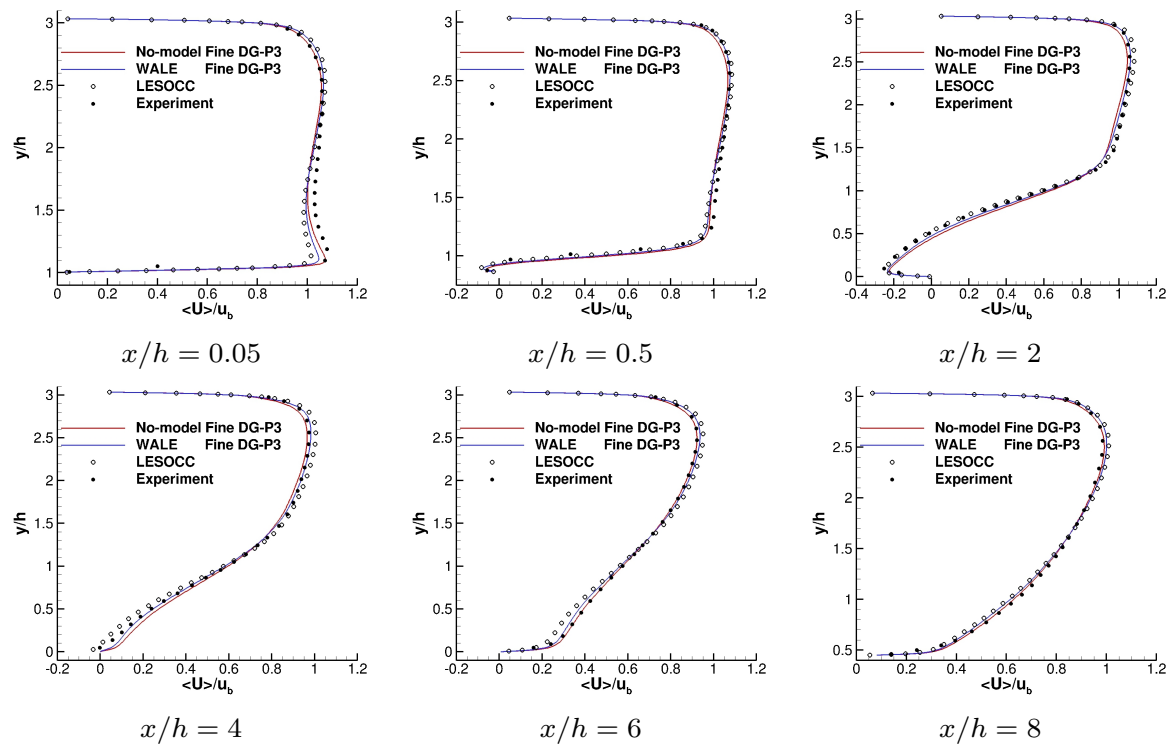

(a) Mean streamwise velocity $U / u_{b}$ profiles
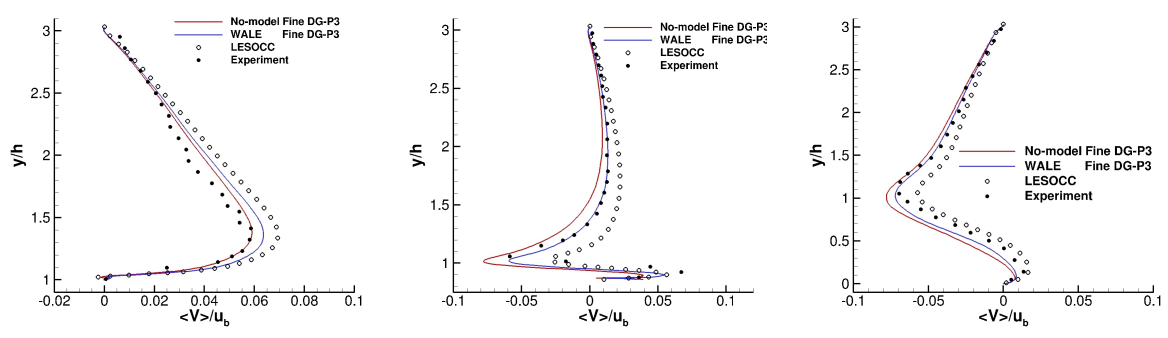

$x / h=0.05$

$x / h=0.5$
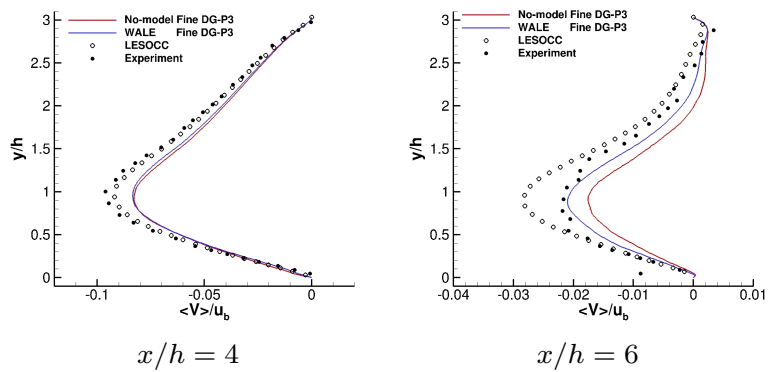

$x / h=2$

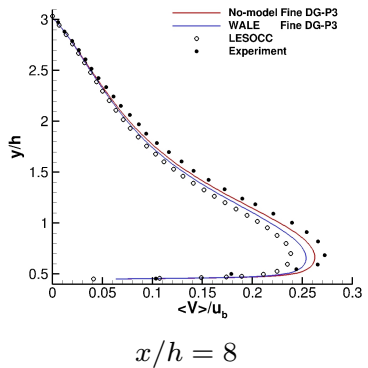

(b) Mean vertical velocity $V / u_{b}$ profiles

Figure 12: LES at $R e_{b}=10595$. Comparison of DG simulations with reference data. • Experiment; o : LESOCC $O(2)$ (13.1 M dofs); - : No-model $D G-p 3$ fine $(4.19 \mathrm{M}$ dofs); : WALE $D G-p 3$ fine $(4.19 \mathrm{M}$ dofs $)$. 

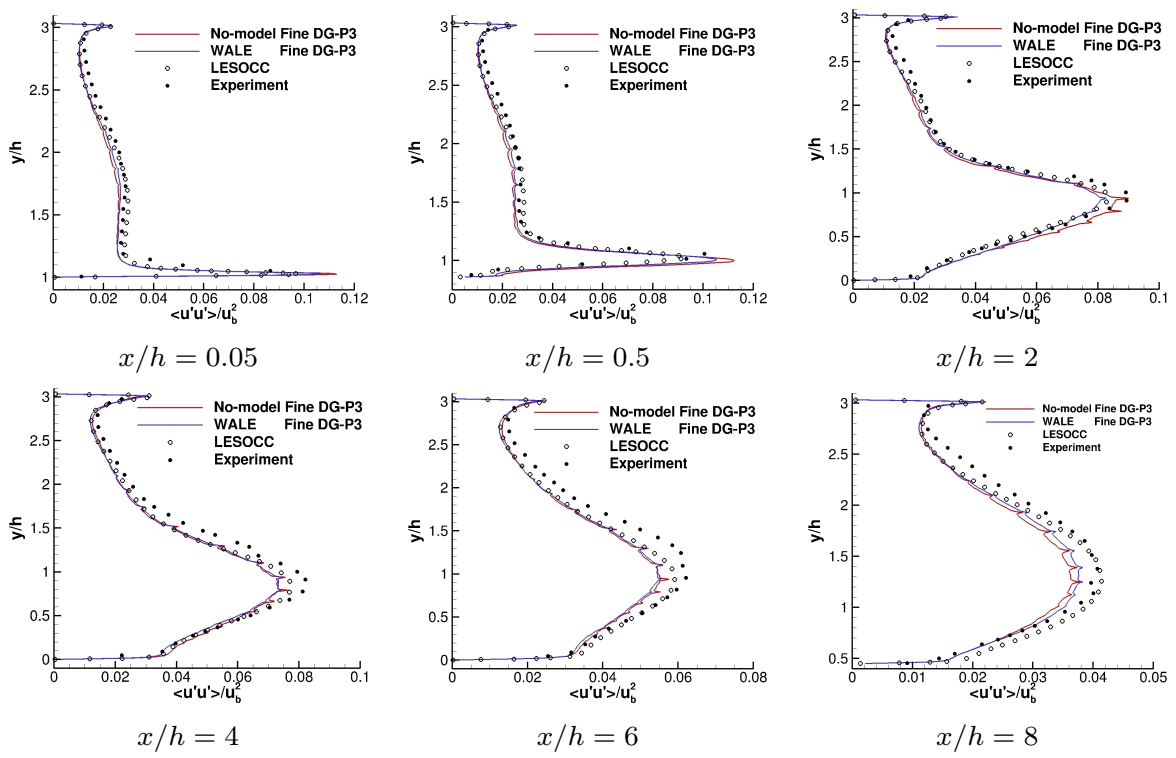

(a) Reynolds stress $\left\langle u^{\prime} u^{\prime}\right\rangle / u_{b}^{2}$ profiles
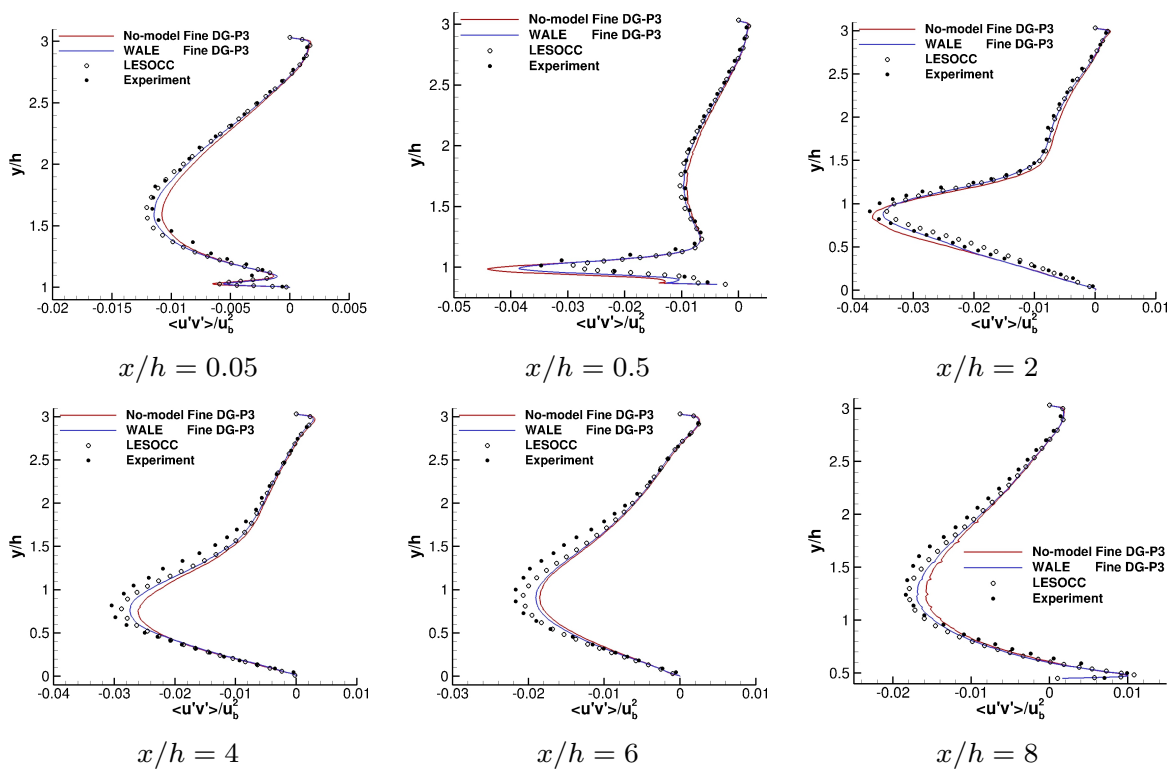

(b) Shear stress $\left\langle u^{\prime} v^{\prime}\right\rangle / u_{b}^{2}$ profiles

Figure 13: LES at $R e_{b}=10595$. Comparison of DG simulations with reference data. - : Experiment; o : LESOCC $O(2)(13.1 \mathrm{M}$ dofs $) ;-$ : No-model $D G-p 3$ fine $(4.19 \mathrm{M} \mathrm{dofs}) ;-$ : WALE $D G-p 3$ fine (4.19 M dofs). 

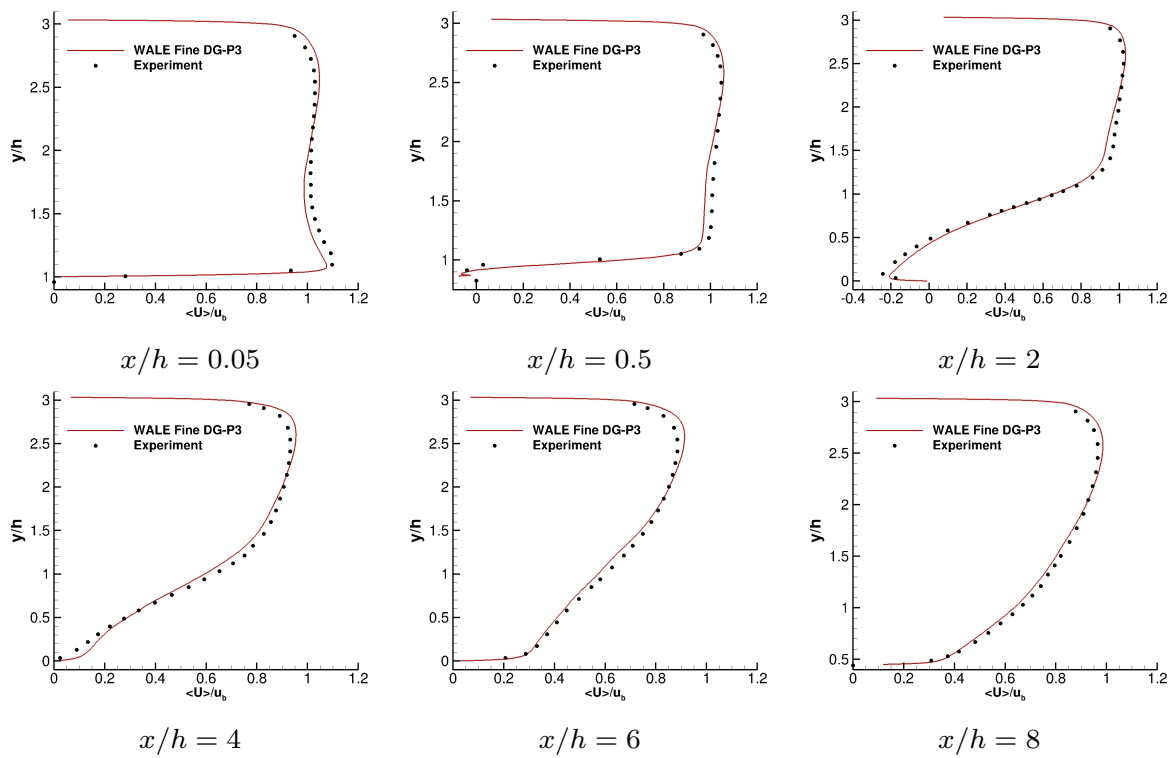

(a) Mean streamwise velocity $U / u_{b}$ profiles
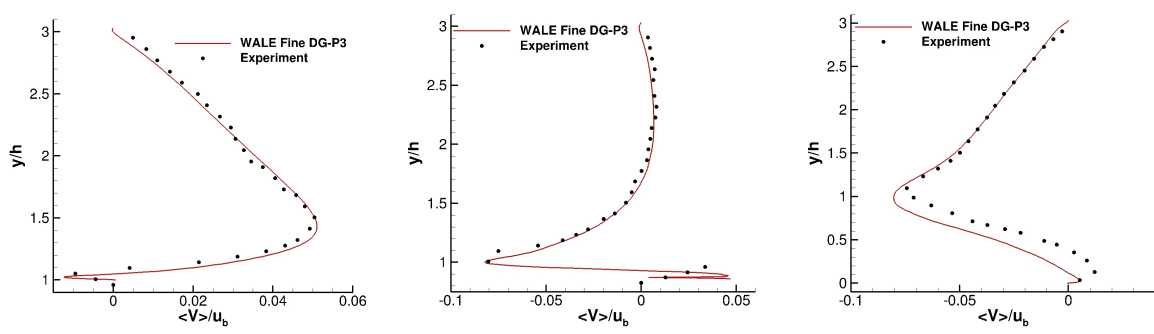

$x / h=0.05$

$x / h=0.5$

$x / h=2$
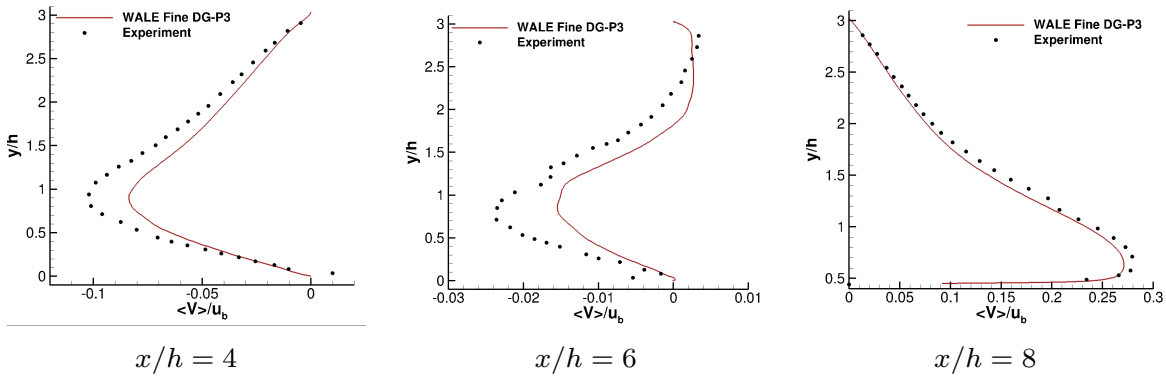

(b) Mean vertical velocity $V / u_{b}$ profiles

Figure 14: LES at $R e=19000$. Comparison of DG simulations with experimental data. • Experiment; - : WALE $D G-p 3$ fine (4.19 Mdofs). 

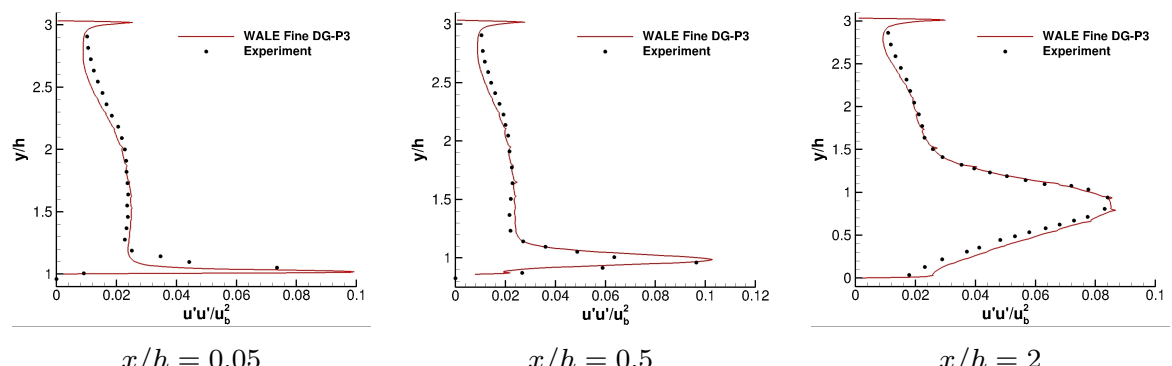

$x / h=0.5$

$x / h=2$
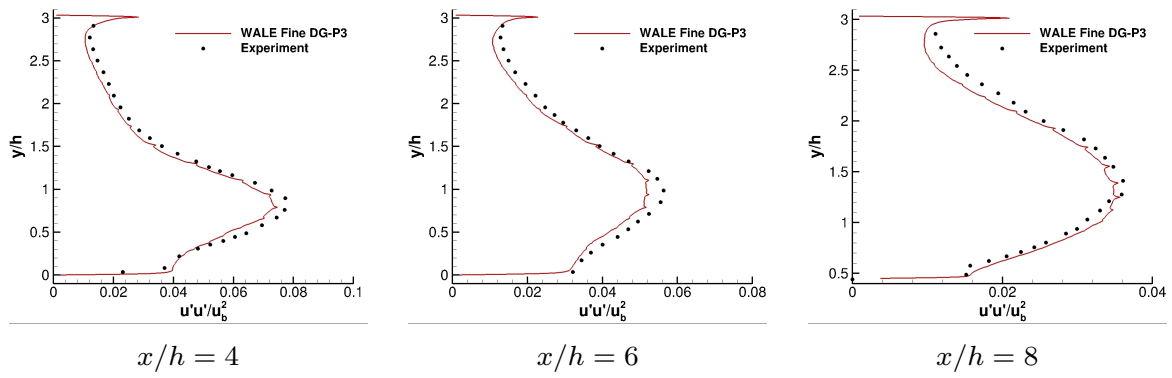

(a) Reynolds stress $\left\langle u^{\prime} u^{\prime}\right\rangle / u_{b}^{2}$ profiles
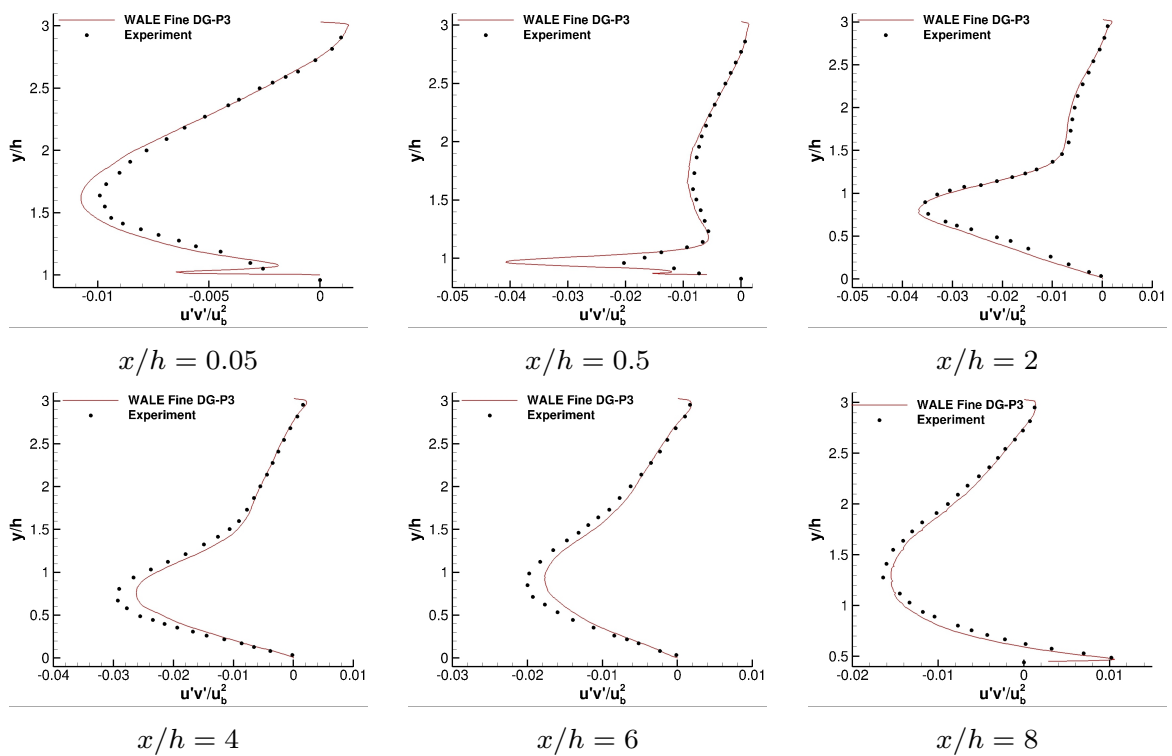

(b) Shear stress $\left\langle u^{\prime} v^{\prime}\right\rangle / u_{b}^{2}$ profiles

Figure 15: LES at $R e=19000$. Comparison of DG simulations with experimental data. • Experiment; - : WALE $D G-p 3$ simulation on fine mesh (4.19 Mdofs). 

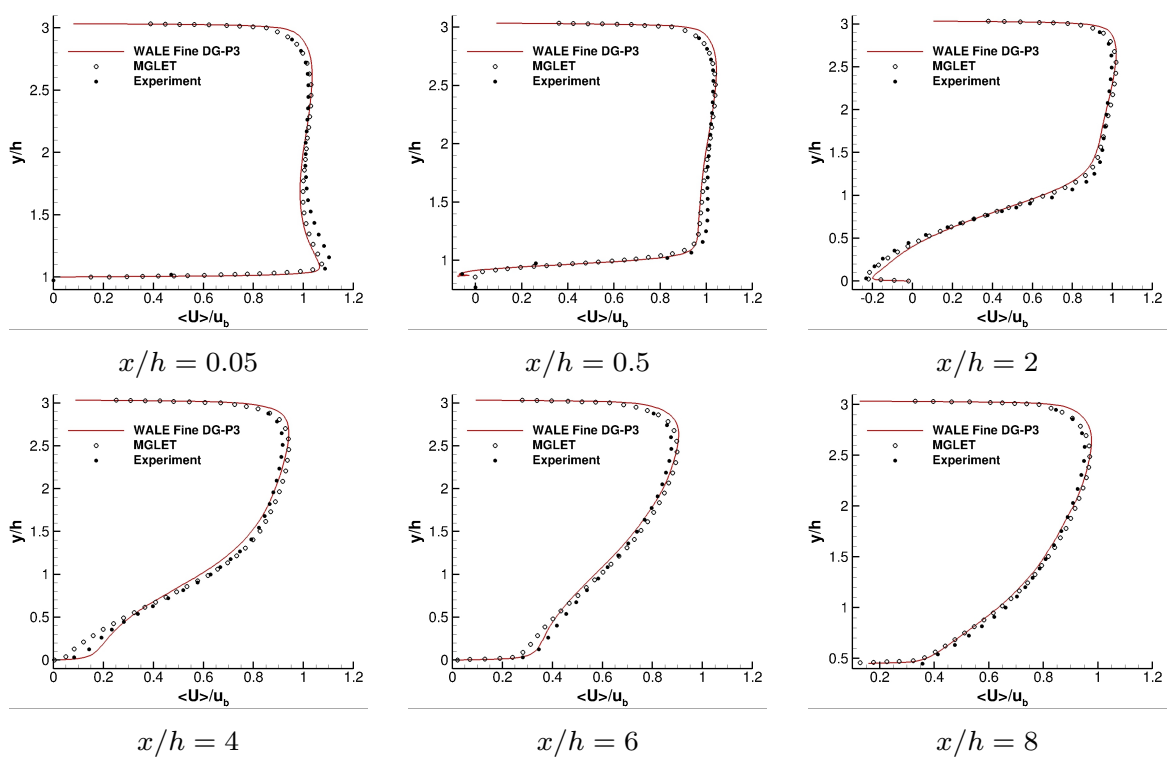

(a) Mean streamwise velocity $U / u_{b}$ profiles
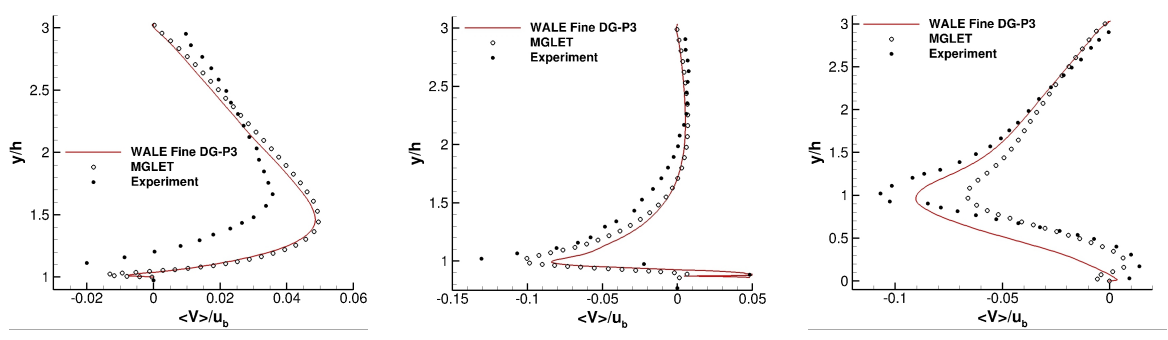

$x / h=0.05$

$x / h=0.5$
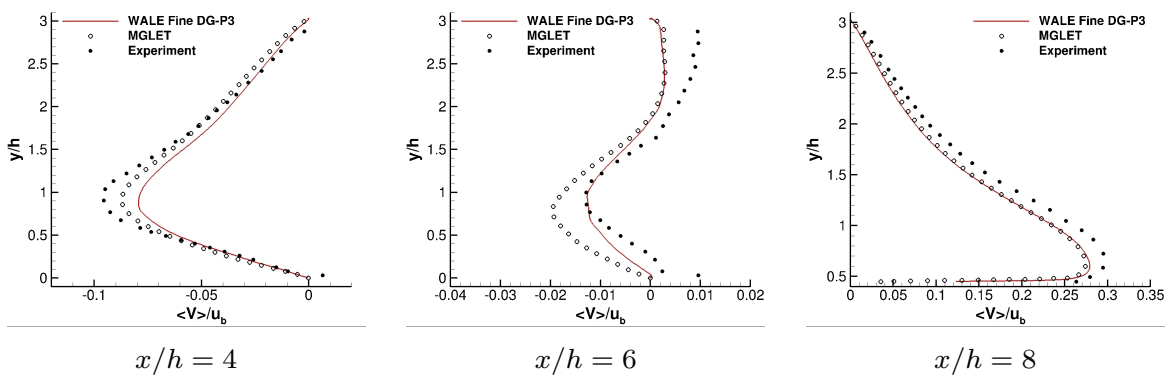

(b) Mean vertical velocity $V / u_{b}$ profiles

Figure 16: LES at $R e=37000$. Comparison of DG simulations with reference MGLET simulation and experimental data. - : Experiment; $\circ$ : MGLET $O(2)$ (4.1 Mdofs); - : WALE $D G-p 3$ fine (4.19 Mdofs). 

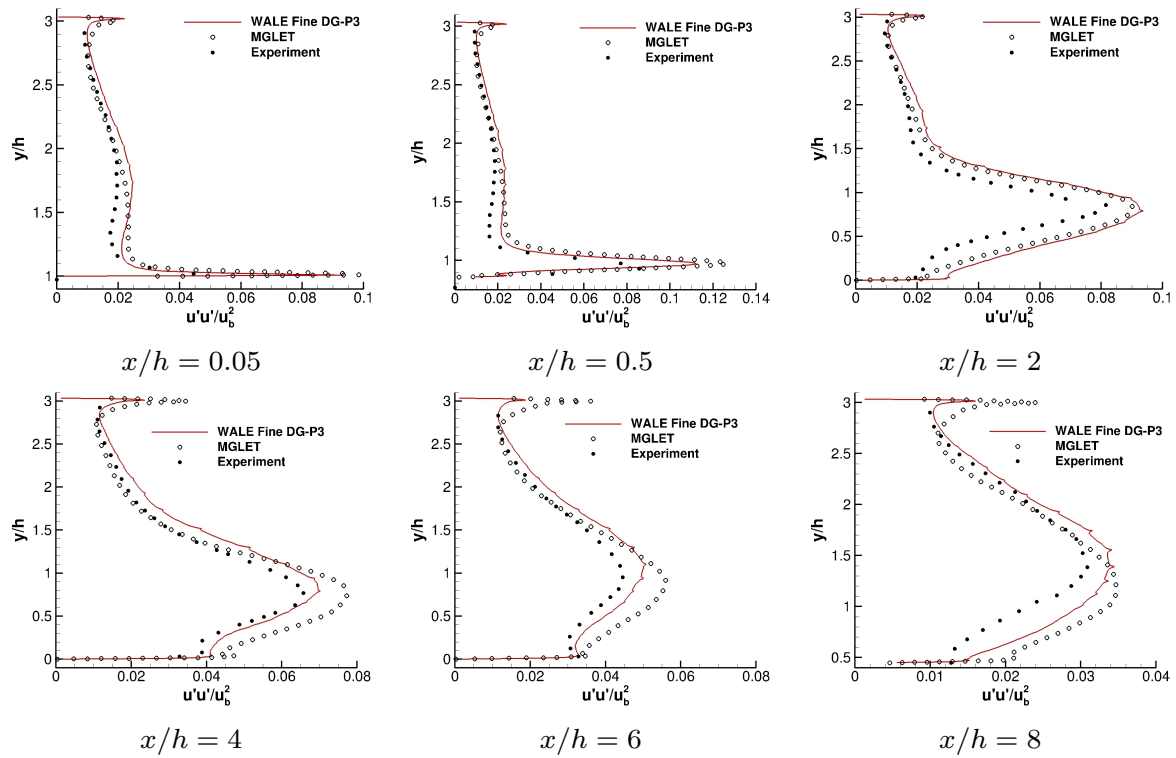

(a) Reynolds stress $\left\langle u^{\prime} u^{\prime}\right\rangle / u_{b}^{2}$ profiles
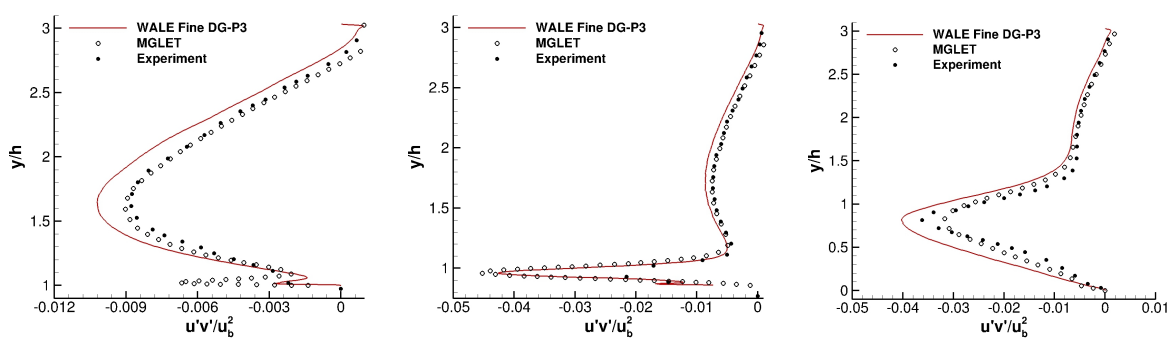

$x / h=0.05$

$x / h=0.5$

$x / h=2$
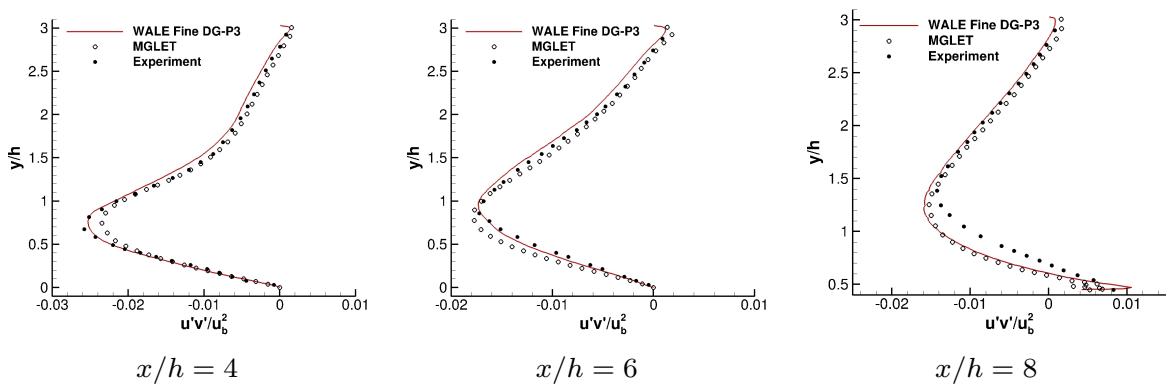

(b) Shear stress $\left\langle u^{\prime} v^{\prime}\right\rangle / u_{b}^{2}$ profiles

Figure 17: LES at $R e=37000$. Comparison of DG simulations with reference MGLET simulation and experimental data. - : Experiment; $\circ$ : MGLET $O(2)$ (4.1 Mdofs); - : WALE $D G-p 3$ fine (4.19 Mdofs). 